\title{
ANALYSIS AND APPROXIMATION OF THE VELOCITY TRACKING PROBLEM FOR NAVIER-STOKES FLOWS WITH DISTRIBUTED CONTROL*
}

\author{
M. D. GUNZBURGER ${ }^{\dagger}$ AND S. MANSERVISI ${ }^{\ddagger}$
}

\begin{abstract}
We consider the mathematical formulation, analysis, and the numerical solution of a time-dependent optimal control problem associated with the tracking of the velocity of a NavierStokes flow in a bounded two-dimensional domain through the adjustment of a distributed control. The existence of optimal solutions is proved and the first-order necessary conditions for optimality are used to derive an optimality system of partial differential equations whose solutions provide optimal states and controls. Semidiscrete-in-time and fully discrete space-time approximations are defined and their convergence to the exact optimal solutions is shown. A gradient method for the solution of the fully discrete equations is examined, as are its convergence properties. Finally, the results of some illustrative computational experiments are presented.
\end{abstract}

Key words. optimal control, Navier-Stokes equations, finite elements, fluid mechanics

AMS subject classifications. 35B40, 35B37, 35Q30, 65M60

PII. S0036142997329414

1. Introduction. The purpose of the velocity tracking problem is to steer, over time, a velocity field to a given target velocity field. In this paper, we consider controls that act as a distributed body force; the state of the system, i.e., the velocity and pressure fields, is the solution of an initial-boundary value problem of the Navier-Stokes system of partial differential equations that models the evolution of viscous, incompressible flows. The cost or objective functional is a quadratic functional involving the state and the control variables; the functional measures, in an appropriate norm, the distance between the flow velocity and the target velocity fields, and through a penalty term, also measures the cost of control. Thus, the minimization of the functional is used to both drive the flow towards the target flow and to limit the cost of control.

Several treatments of similar optimal control problems can be found in the literature, most notably in [1], [5], [6], and [7]. Our work differs from these in that we use a different functional that we show does a better job of tracking the target velocity field; also, compared to previous authors, we use general, e.g., nonseparable, controls and weaker hypotheses on the domain. The numerical treatment of the velocity tracking problem is also an outstanding problem and other algorithms have been proposed. For example, a quasi-optimal control has been studied in [12] and [13].

In this paper, we will formulate the problem in a convenient and precise mathematical way; then, we will prove the existence of optimal controls and characterize optimal controls by deriving the first-order necessary conditions associated with the problem. We then examine semidiscrete-in-time and fully discrete in space and time

* Received by the editors October 30, 1997; accepted for publication (in revised form) August 11, 1999; published electronically May 4, 2000.

http://www.siam.org/journals/sinum/37-5/32941.html

${ }^{\dagger}$ Department of Mathematics, Iowa State University, Ames, IA 50011-2064 (gunzburg@ iastate.edu). This author was supported in part by the Air Force Office of Scientific Research under grant F49620-95-1-0407.

${ }^{\ddagger}$ Department of Physics, Virginia Tech, Blacksburg, VA 24061-0435. Current address: ITWM-Mathematics Department, University of Kaiserslautern, D-67663 Kaiserslautern, Germany (manservisi@itwm.uni-kl.de). 
finite element based approximations of solutions of the optimality system and develop a gradient algorithm for the solution of the discrete equations. A frontal method for assembling and solving the finite element system is used.

The plan of the rest of the paper is as follows. In section 2, we will treat the continuous optimal control problem. In section 3 and section 4, we will analyze semidiscrete and the fully discrete approximations, respectively. In section 5 , a gradient method for the solution of the fully discrete optimal control problem is presented and analyzed. Finally, in section 6 , the results of some computational experiments are presented.

\section{The optimal control problem.}

2.1. Notation and formulation of the optimal control problem. Let $\Omega$ be a bounded open set. We shall use the standard notations for the Sobolev spaces $H^{m}(\Omega)$ with norm $\|\cdot\|_{m}\left(H^{0}(\Omega)=L^{2}(\Omega)\right.$ and $\left.\|\cdot\|_{0}=\|\cdot\|\right)$ and their vector-valued $\left(\mathbb{R}^{2}\right.$-valued) counterparts for which we use the same notation. Let $H_{0}^{m}(\Omega)$ denote the closure of $C_{0}^{\infty}(\Omega)$ under the norm $\|\cdot\|_{m}$ and $H^{-m}(\Omega)$ be the dual space of $H_{0}^{m}(\Omega)$. Also, we define

$$
L_{0}^{2}(\Omega)=\left\{p \in L^{2}(\Omega): \int_{\Omega} p d \vec{x}=0\right\} .
$$

For details concerning these spaces, see, e.g., [2] or [8]. We introduce the solenoidal spaces

$$
\begin{aligned}
& \mathcal{V}(\Omega)=\left\{\vec{u} \in C_{0}^{\infty}(\Omega): \nabla \cdot \vec{u}=0\right\}, \\
& V(\Omega)=\left\{\vec{u} \in H_{0}^{1}(\Omega): \nabla \cdot \vec{u}=0\right\}, \\
& W(\Omega)=\left\{\vec{u} \in L^{2}(\Omega): \nabla \cdot \vec{u}=0\right\} .
\end{aligned}
$$

The spaces $W(\Omega)$ and $V(\Omega)$ are the closures of $\mathcal{V}(\Omega)$ in $L^{2}(\Omega)$ and $H_{0}^{1}(\Omega)$, respectively. The dual space of $V(\Omega)$ is denoted by $V(\Omega)^{*}$ while the dual of $W(\Omega)$ can be identified with itself. Given $T$, we introduce the notation $L^{p}((0, T) ; X)$ for the temporal-spatial function spaces defined on $(0, T) \times \Omega$ with the norm

$$
\|\vec{u}\|_{L^{p}((0, T) ; X)}=\left(\int_{0}^{T}\|\vec{u}\|_{X}^{p} d t\right)^{1 / p} .
$$

In order to define a weak form of the Navier-Stokes equations, we introduce the continuous bilinear forms

$$
\begin{aligned}
& a(\vec{u}, \vec{v})=2 \nu \sum_{i, j=1}^{2} \int_{\Omega} D_{i j}(\vec{u}) D_{i j}(\vec{v}) d \vec{x} \quad \forall \vec{u}, \vec{v} \in H^{1}(\Omega), \\
& b(\vec{v}, q)=-\int_{\Omega} q \vec{\nabla} \cdot \vec{v} d \vec{x} \quad \forall q \in L_{0}^{2} \quad \forall \vec{v} \in H^{1}(\Omega),
\end{aligned}
$$

where $D_{i j}(\vec{u})=\left(\partial u_{i} / \partial x_{j}+\partial u_{j} / \partial x_{i}\right) / 2$, and the trilinear form

$$
c(\vec{w} ; \vec{u}, \vec{v})=\sum_{i, j=1}^{2} \int_{\Omega} w_{j}\left(\frac{\partial u_{i}}{\partial x_{j}}\right) v_{i} d \vec{x} \quad \forall \vec{w}, \vec{u}, \vec{v} \in H^{1}(\Omega) .
$$

In (2.1), $\nu$ denotes the inverse of the Reynolds number whenever the variables are appropriately nondimensionalized. A weak formulation of the Navier-Stokes problem is given by 
given $\vec{g} \in H^{-1}(\Omega)$, find $(\vec{u}, p) \in H_{0}^{1}(\Omega) \times L_{0}^{2}(\Omega)$ satisfying

$$
\begin{aligned}
& \left\langle\frac{\partial \vec{u}}{\partial t}, \vec{v}\right\rangle+a(\vec{u}, \vec{v})+c(\vec{u} ; \vec{u}, \vec{v})+b(\vec{u}, p)=\langle\vec{g}, \vec{v}\rangle \quad \forall v \in H_{0}^{1}(\Omega), \\
& b(\vec{u}, q)=0 \quad \forall q \in L_{0}^{2}(\Omega)
\end{aligned}
$$

with initial velocity $\vec{u}_{0}$,

where $\langle\cdot, \cdot\rangle$ denotes the duality pairing between $H_{0}^{1}(\Omega)$ and $H^{-1}(\Omega)$. Since $\vec{u} \in H_{0}^{1}(\Omega)$, we see that we have homogeneous boundary conditions for $\vec{u}$.

Let us define the operators $A: H_{0}^{1}(\Omega) \rightarrow H^{-1}(\Omega), B: H_{0}^{1}(\Omega) \rightarrow L_{0}^{2}(\Omega)$, and $C: H_{0}^{1}(\Omega) \times H_{0}^{1}(\Omega) \rightarrow H^{-1}(\Omega)$ in the usual way (see $[8]$ ):

$$
\begin{aligned}
& \langle A \vec{u}, \vec{v}\rangle=a(\vec{u}, \vec{v}) \quad \forall \vec{u}, \vec{v} \in H_{0}^{1}(\Omega), \\
& \langle C(\vec{w}) \vec{u}, \vec{v}\rangle=c(\vec{w} ; \vec{u}, \vec{v}) \quad \forall \vec{u}, \vec{v}, \vec{w} \in H_{0}^{1}(\Omega), \\
& (q, B \vec{v})=b(\vec{v}, q) \quad \forall \vec{v} \in H_{0}^{1}(\Omega) \quad \forall q \in L_{0}^{2}(\Omega), \\
& \left\langle B^{*} q, \vec{v}\right\rangle=b(\vec{v}, q) \quad \forall \vec{v} \in H_{0}^{1}(\Omega) \quad \forall q \in L_{0}^{2}(\Omega),
\end{aligned}
$$

where $B^{*}$ denotes the dual operator and where $(\cdot, \cdot)$ denotes the inner product in $L^{2}(\Omega)$. Since the boundary conditions are homogeneous Dirichlet boundary conditions, we can choose the test function in (2.4) to belong to $V(\Omega)$ and then the linear form $b(\vec{v}, p)$ vanishes. As a result, we have that (2.4) can be expressed in the form

$$
\frac{d \vec{u}}{d t}+A \vec{u}+C(\vec{u}) \vec{u}=\vec{g}, \quad \vec{u} \in V(\Omega) .
$$

Every function in $\mathcal{D}^{\prime}(\Omega)$ orthogonal to $\mathcal{V}(\Omega)$, which is dense in $V(\Omega)$, can be written as a gradient of some $p$ in $\mathcal{D}^{\prime}(\Omega)$. The equivalence with the classical problem is now clear. For details concerning the notation employed and the properties of the bilinear and trilinear forms one may consult, e.g., [8], [9], or [14].

We shall consider only target velocity fields $\vec{U} \in \mathcal{U}_{a d}$, where $\mathcal{U}_{a d}$ is defined by

$$
\left\{\begin{array}{l}
\vec{U}=\vec{U}(t, \vec{x}) \in C\left([0, T] ; H^{2}(\Omega)\right), \\
\vec{\nabla} \cdot \vec{U}(t, \vec{x})=0 \quad \forall \vec{x} \in \Omega, \\
\vec{U}(t, \vec{x})=0 \quad \forall \vec{x} \in \Gamma \\
\vec{F}_{\vec{U}}(t, \vec{x}) \in L^{\infty}\left((0, T) ; L^{2}(\Omega)\right),
\end{array}\right.
$$

where $\vec{F}_{\vec{U}}=\vec{U}_{t}-\nu \nabla^{2} \vec{U}+(\vec{U} \cdot \vec{\nabla}) \vec{U}$. The divergence free constraint and the homogeneous boundary condition for the target velocity field are not necessary for the mathematical treatment of the problem, but they are assumed in order to give a physical meaning to the target flow.

Let $\vec{g} \in L^{2}\left((0, T) ; L^{2}(\Omega)\right)$ denote the distributed control. Given $T$, we define the functional

$$
\mathcal{J}(\vec{u}, \vec{g})=\int_{0}^{T} \int_{\Omega}\left(\frac{\alpha}{2}|\vec{u}-\vec{U}|^{2}+\frac{\beta}{2}|\vec{g}|^{2}\right) d \vec{x} d t+\frac{\gamma}{2} \int_{\Omega}|\vec{u}(T)-\vec{U}(T)|^{2} d \vec{x} .
$$

The minimization of the $\int_{0}^{T} \int_{\Omega}|\vec{u}-\vec{U}|^{2} d \vec{x} d t$ term is the real goal of the velocity tracking problem, which is to keep the solution $\vec{u}$ close to $\vec{U}$ over $(0, T) \times \Omega$. The 
$\int_{0}^{T} \int_{\Omega}|\vec{g}|^{2} d \vec{x} d t$ term is introduced in order to bound the control function and to prove the existence of an optimal control. The $\int_{\Omega}|\vec{u}(T, \vec{x})-\vec{U}(T, \vec{x})|^{2} d \vec{x}$ term is necessary in order to keep the solution $\vec{u}$ close to $\vec{U}$ near the time $T$. This term is not included in previous studies of the velocity tracking problem; its omission results in poorer performance of the optimal control.

The set of all admissible solutions is denoted by $\mathcal{A}_{a d}$ and is defined as follows.

Given $\Omega, T, \vec{u}_{0} \in V(\Omega)$, and $\vec{U} \in \mathcal{U}_{a d}$, then $(\vec{u}, \vec{g})$ is said to be an admissible element if $\left.\vec{u} \in L^{2}(0, T) ; H_{0}^{1}(\Omega)\right), \vec{g} \in L^{2}\left((0, T) ; L^{2}(\Omega)\right)$, the functional $\mathcal{J}(\vec{u}, \vec{g})$ is bounded, and there exists a $\left.p \in L^{2}(0, T) ; L_{0}^{2}(\Omega)\right)$ such that $(\vec{u}, p, \vec{g})$ satisfies (2.4) and (2.5).

With this notation, the formulation of the optimal control problem is given by

$$
\begin{gathered}
\text { given } \Omega, T, \vec{u}_{0} \in V(\Omega) \text {, and } \vec{U} \in \mathcal{U}_{a d}, \text { find }(\vec{u}, \vec{g}) \in \mathcal{A}_{a d} \text { such that } \\
\qquad \mathcal{J}(\vec{u}, \vec{g}) \leq \mathcal{J}(\vec{w}, \vec{h}) \quad \forall(\vec{w}, \vec{h}) \in \mathcal{A}_{a d} .
\end{gathered}
$$

2.2. Existence of an optimal control solution. We recall that if $\Gamma$ is Lipschitz continuous, $\vec{g} \in L^{2}\left((0, T) ; H^{-1}(\Omega)\right)$, and $\vec{u}_{0} \in W(\Omega)$, then solutions of $(2.4)$ and (2.5) satisfy $\vec{u} \in C([0, T] ; W(\Omega)) \cap L^{2}((0, T) ; V(\Omega))$ and $\vec{u}_{t} \in L^{2}\left((0, T) ; H^{-1}(\Omega)\right)$. If $\Gamma \in C^{2}, \vec{u}_{0} \in V(\Omega)$, and $\vec{g} \in L^{2}((0, T) ; W(\Omega))$, then $\vec{u} \in C([0, T] ; V(\Omega)) \cap$ $L^{2}\left((0, T) ; H^{2} \cap H_{0}^{1}\right)$ and $\vec{u}_{t} \in L^{2}((0, T) ; W(\Omega))$. See, e.g., [4] or [14].

We prove the existence of a solution of the optimal control problem when $\Omega$ is an open bounded domain with a Lipschitz continuous boundary $\Gamma$.

Theorem 2.1. Given $\vec{u}_{0} \in V(\Omega)$ and $\vec{U} \in \mathcal{A}_{a d}$, then there exists a solution $\vec{g} \in L^{2}((0, T) ; W(\Omega))$ and $\vec{u} \in C([0, T] ; W(\Omega)) \cap L^{2}((0, T) ; V(\Omega))$ of the optimal control problem.

Proof. As the admissible set is bounded and not empty, e.g., $(\vec{u}, 0) \in \mathcal{A}_{a d}$, let $\vec{g}_{n}$ be a minimizing sequence for the optimal control problem and set $\vec{u}_{n}=\vec{u}\left(\vec{g}_{n}\right)$. The sequence $\left\{\vec{g}_{n}\right\}$ is bounded in $L^{2}((0, T) ; W(\Omega))$ and the corresponding solution $\vec{u}_{n}$ is bounded in $C([0, T] ; W(\Omega)) \cap L^{2}((0, T) ; V(\Omega))$. This follows from well-known existence theorems for solutions of the unsteady Navier-Stokes equations; see [14]. As Hilbert spaces are reflexive, every ball is weakly compact and thus there exist a pair $(\vec{u}, \vec{g})$ and a subsequence of $\left(\vec{u}_{n}, \vec{g}_{n}\right)$ that converges weakly to $(\vec{u}, \vec{g})$. We abuse the notation and write again that

$$
\begin{array}{llll}
\vec{g}_{n} \rightarrow \vec{g} & \text { in } & L^{2}((0, T) ; W(\Omega)) & \text { weakly, } \\
\vec{u}_{n} \rightarrow \vec{u} & \text { in } & L^{2}((0, T) ; V(\Omega)) & \text { weakly, } \\
\vec{u}_{n} \rightarrow \vec{u} & \text { in } & L^{\infty}((0, T) ; W(\Omega)) & \text { weak }-* .
\end{array}
$$

Now, the pair $(\vec{u}, \vec{g})$ satisfies the Navier-Stokes equations (2.4) and (2.5) and minimizes the functional. In fact, by the lower semicontinuity of the functional in (2.8) we have

$$
\mathcal{J}(\vec{u}, \vec{g}) \leq \liminf _{n \rightarrow \infty} \mathcal{J}\left(\vec{u}_{n}, \vec{g}_{n}\right) .
$$

Besides, an a priori estimate (see [4] or [14]) for $\vec{u}$ in a fractional time order Sobolev space yields, in our case, that $\vec{u}_{n}$ converges strongly to $\vec{u} \in L^{2}((0, T) ; V(\Omega))$. Now, we consider the weak Navier-Stokes system given by (2.6) with state $\vec{u}_{n}$ and control $\vec{g}_{n}$. Let $\vec{w}$ be in $\mathcal{V}(\Omega)$ and $\psi(t)$ be a continuously differentiable function on $(0, T)$ with 
$\psi(T)=0$. We multiply (2.6) by $\psi(\tau) \vec{w}$ and then integrate by parts with respect to $\tau$ to obtain

$$
\begin{gathered}
-\int_{0}^{T}\left(\vec{u}_{n}, \psi^{\prime}(\tau) \vec{w}\right) d \tau+\nu \int_{0}^{T} a\left(\vec{u}_{n}, \psi(\tau) \vec{w}\right) d \tau+\int_{0}^{T} c\left(\vec{u}_{n} ; \vec{u}_{n}, \psi(\tau) \vec{w}\right) d \tau \\
=\left(\vec{u}_{0}, \psi(0) \vec{w}\right)+\int_{0}^{T}\left(\vec{g}_{n}, \psi(\tau) \vec{w}\right) d \tau
\end{gathered}
$$

We can pass to the limit inside the linear and the nonlinear terms. In fact, if $\vec{u}_{n}$ converges to $\vec{u}$ in $L^{2}((0, T) ; V(\Omega))$ weakly and $L^{2}((0, T) ; W(\Omega))$ strongly, then, for any $\vec{z} \in C^{1}((0, T) ; \mathcal{D}(\Omega))$, we have

$$
\lim _{n \rightarrow \infty} \int_{0}^{T} c\left(\vec{u}_{n} ; \vec{u}_{n}, \vec{z}(\tau)\right) d \tau=\int_{0}^{T} c(\vec{u} ; \vec{u}, \vec{z}(\tau)) d \tau ;
$$

see [14]. If $\psi=\phi \in \mathcal{D}(0, T)$, the pair $(\vec{u}, \vec{g})$ satisfies the Navier-Stokes equation (2.6) in the distributional sense. Since $\mathcal{V}(\Omega)$ is dense in $V(\Omega)$, then this is still true for any $\vec{w}$ in $V(\Omega)$ by a continuity argument.

2.3. First-order necessary condition. We shall show that the optimal solution must satisfy the first-order necessary condition. By studying the case in which the Gâteaux derivative vanishes we can get a possible candidate solution for the optimal control. Now, it is very useful to state here some results concerning the differentiability of the operator $C$. The proof of this lemma can be found in [1].

Lemma 2.2. Let $c(\vec{w} ; \vec{u}, \vec{v})$ be the trilinear form defined in (2.3) and let $C(\vec{w}) \vec{u}$ denote the corresponding operator. Then,

(i) $c(\vec{w} ; \vec{u}, \vec{v})$ has the following properties $\forall \vec{w}, \vec{u}, \vec{v} \in V(\Omega)$ :

$$
\left\{\begin{array}{l}
c(\vec{w} ; \vec{u}, \vec{v})=-c(\vec{w} ; \vec{v}, \vec{u}), \\
c(\vec{w} ; \vec{u}, \vec{u})=0, \\
|c(\vec{w} ; \vec{u}, \vec{v})| \leq\|\vec{w}\|_{1}\|\vec{u}\|_{1}\|\vec{v}\|_{1}, \\
|c(\vec{w} ; \vec{u}, \vec{v})| \leq \sqrt{2}\|\vec{w}\|^{1 / 2}\|\nabla \vec{w}\|^{1 / 2}\|\nabla \vec{u}\|\|\vec{v}\|^{1 / 2}\|\nabla \vec{v}\|^{1 / 2}
\end{array}\right.
$$

(ii) given $\vec{u} \in V(\Omega), C(\vec{u}) \vec{u}$ is a differentiable form from $V(\Omega)$ into $V^{*}(\Omega)$ and

$$
C^{\prime}(\vec{u}) \vec{b}=C(\vec{u}) \vec{b}+C(\vec{b}) \vec{u} \quad \forall \vec{b} \in V(\Omega) ;
$$

(iii) the adjoint $C^{* *}$ of $C^{\prime}$, defined by $c^{\prime}(\vec{a} ; \vec{b}, \vec{c})=\left(\vec{b}, C^{*}(\vec{a}) \vec{c}\right)$, is given by

$$
\left(C^{\prime *}(\vec{a}) \vec{b}, \vec{c}\right)=\int_{\Omega} \sum_{i, j=1}^{2} c_{j}\left(\frac{\partial a_{i}}{\partial x_{j}} b_{i}-a_{i} \frac{\partial b_{j}}{\partial x_{i}}\right) d \vec{x} .
$$

By using this lemma, we are now ready to state and prove the existence of the Gâteaux derivative. It is useful to remark that the Gâteaux derivative makes sense whenever one is able to prove the uniqueness of the solution of the Navier-Stokes system. In the time-dependent, two-dimensional case, this is possible.

Theorem 2.3. Let $\vec{u}_{0} \in V(\Omega)$. The mapping $\vec{u}=\vec{u}(\vec{g})$ from $L^{2}\left((0, T) ; L^{2}(\Omega)\right)$ to $L^{2}((0, T) ; V(\Omega))$, defined as the solution of $(2.6)$, has a Gâteaux derivative $(D \vec{u} / D \vec{g}) \cdot \vec{h}$ 
in every direction $\vec{h}$ in $L^{2}\left((0, T) ; L^{2}(\Omega)\right)$. Furthermore, $\widetilde{w}(h)=(D \vec{u} / D \vec{g}) \cdot \vec{h}$ is the solution of the problem

$$
\left\{\begin{array}{l}
\widetilde{w}_{t}+\nu A \widetilde{w}+C(\vec{u}(\vec{g})) \widetilde{w}+C(\widetilde{w}) \vec{u}(\vec{g})=\vec{h} \\
\widetilde{w} \in V(\Omega), \\
\widetilde{w}(t, \vec{x})=0, \quad \vec{x} \in \Gamma \\
\widetilde{w}(0, \vec{x})=0, \quad \vec{x} \in \Omega
\end{array}\right.
$$

Moreover, $\widetilde{w} \in L^{\infty}((0, T) ; W(\Omega)) \cap L^{2}((0, T) ; V(\Omega))$.

Proof. Let $\vec{g}$ and $\vec{h}$ be given in $L^{2}((0, T) ; W(\Omega))$ and let $\vec{u}_{\vec{g}}$ and $\vec{u}_{\vec{g}+s \vec{h}}$ denote the solutions of (2.6) with right-hand sides $\vec{g}$ and $\vec{g}+s \vec{h}$, respectively. We need to prove the following result:

$$
\lim _{s \rightarrow 0}\left(\frac{\left\|\vec{u}_{\vec{g}+s \vec{h}}-\vec{u}_{\vec{g}}-s \widetilde{w}(\vec{h})\right\|_{L^{2}((0, T) ; V)}}{|s|}\right)=0 .
$$

We set $\widetilde{u}=\left(\vec{u}_{\vec{g}+s \vec{h}}-\vec{u}_{\vec{g}}\right)-s \widetilde{w}(\vec{h})$ so that $\widetilde{u}$ is the solution of the evolution equation

$$
\left\{\begin{array}{l}
\frac{d \widetilde{u}}{d t}+\nu A \widetilde{u}+C\left(\vec{u}_{\vec{g}+s \vec{h}}\right) \vec{u}_{\vec{g}+s \vec{h}}-C\left(\vec{u}_{\vec{g}}\right) \vec{u}_{\vec{g}}-C^{\prime}\left(\vec{u}_{\vec{g}}\right) s \widetilde{w}=0 \\
\widetilde{u} \in V(\Omega), \\
\widetilde{u}(t, \vec{x})=0, \quad \vec{x} \in \Gamma \\
\widetilde{u}(0, \vec{x})=0, \quad \vec{x} \in \Omega .
\end{array}\right.
$$

Let $\vec{k} \in H^{-1}(\Omega)$ be defined by

$$
\vec{k}=C\left(\vec{u}_{\vec{g}}\right) \vec{u}_{\vec{g}}+C^{\prime}\left(\vec{u}_{\vec{g}}\right)\left(\vec{u}_{\vec{g}+s \vec{h}}-\vec{u}_{\vec{g}}\right)-C\left(\vec{u}_{\vec{g}+s \vec{h}}\right) \vec{u}_{\vec{g}+s \vec{h}} ;
$$

then, (2.15) can be rewritten as

$$
\left\{\begin{array}{l}
\frac{d \widetilde{u}}{d t}+\nu A \widetilde{u}+C^{\prime}\left(\vec{u}_{\vec{g}}\right) \widetilde{u}=\vec{k} \\
\widetilde{u} \in V(\Omega), \\
\widetilde{u}(t, \vec{x})=0, \quad \vec{x} \in \Gamma \\
\widetilde{u}(0, \vec{x})=0, \quad \vec{x} \in \Omega
\end{array}\right.
$$

In order to estimate the norm of $\widetilde{u}$, we take the scalar product of the first equation in (2.17) with $\widetilde{u}$, integrate over $\Omega$, and use part (ii) of Lemma 2.2 in order to obtain

$$
\frac{d}{d t}\|\widetilde{u}\|^{2}+\nu\|\nabla \widetilde{u}\|^{2}+c\left(\widetilde{u} ; \vec{u}_{\vec{g}}, \widetilde{u}\right)=(\vec{k}, \widetilde{u}) .
$$

By using standard techniques and the fourth inequality in part (i) of Lemma 2.2 we have

$$
\int_{0}^{T}\|\widetilde{u}\|_{1}^{2} d t \leq C_{1} \int_{0}^{T}\|\vec{k}\|_{V^{*}}^{2} d t
$$


Now we need to evaluate the right-hand side term above. By taking the scalar product of (2.16) with $\vec{v} \in H_{0}^{1}(\Omega)$, we have

$$
\begin{aligned}
& |<\vec{k}, \vec{v}>|=\left|\left(C\left(\vec{u}_{\vec{g}+s \vec{h}}\right) \vec{u}_{\vec{g}+s \vec{h}}, \vec{v}\right)-\left(C\left(\vec{u}_{\vec{g}}\right) \vec{u}_{\vec{g}}, \vec{v}\right)-\left(C^{\prime}\left(\vec{u}_{\vec{g}}\right) \widehat{u}, \vec{v}\right)\right| \\
& \quad=\left|c\left(\vec{u}_{\vec{g}+s \vec{h}}, \vec{u}_{\vec{g}+s \vec{h}}, \vec{v}\right)-c\left(\vec{u}_{\vec{g}}, \vec{u}_{\vec{g}}, \vec{v}\right)-c\left(\vec{u}_{\vec{g}}, \widehat{u}, \vec{v}\right)-c\left(\widehat{u}, \vec{u}_{\vec{g}}, \vec{v}\right)\right| \\
& \quad=|c(\widehat{u}, \widehat{u}, \vec{v})| \leq|c(\widehat{u}, \vec{v}, \widehat{u})| \leq K\|\widehat{u}\|\|\nabla \widehat{u}\|\|\vec{v}\|_{1},
\end{aligned}
$$

where $\widehat{u}=\vec{u}_{\vec{g}+s \vec{h}}-\vec{u}_{\vec{g}}$. Hence, we obtain

$$
\int_{0}^{T}\|\widetilde{u}(t)\|_{1}^{2} d t \leq C \int_{0}^{T}\|\widehat{u}\|^{2}\|\widehat{u}\|_{1}^{2} d t
$$

To estimate the norm of $\widehat{u}$ in $L^{2}((0, T) ; V(\Omega))$, we again set $\widehat{u}=\vec{u}_{\vec{g}+s \vec{h}}-\vec{u}_{\vec{g}}$ so that

$$
\left\{\begin{array}{l}
\frac{d \widehat{u}}{d t}+\nu A \widehat{u}+C\left(\vec{u}_{\vec{g}}\right) \widehat{u}+C(\widehat{u}) \vec{u}_{\vec{g}}+C(\widehat{u}) \widehat{u}=s \vec{h}, \\
\widehat{u}(t, \vec{x})=0, \quad \vec{x} \in \Gamma, \\
\widehat{u}(0, \vec{x})=0, \quad \vec{x} \in \Omega .
\end{array}\right.
$$

We then easily obtain

$$
\frac{d}{d t}\|\widehat{u}\|^{2}+\nu\|\nabla \widehat{u}\|^{2}+c\left(\widehat{u} ; \vec{u}_{\vec{g}}, \widehat{u}\right)=s(\vec{h}, \widehat{u}) .
$$

Again, by using standard techniques and the fourth inequality of part (i) of Lemma 2.2 , we obtain that $\widehat{u} \in L^{\infty}((0, T) ; W(\Omega))$ (in fact, from the Gronwall inequality we obtain $\|\widehat{u}\| \leq s C(t)$, where $C(t)$ is bounded) and

$$
\int_{0}^{T}\|\widehat{u}\|_{1}^{2} d \tau=\int_{0}^{T}\left\|\vec{u}_{\vec{g}+s \vec{h}}-\vec{u}_{\vec{g}}\right\|_{1}^{2} d \tau \leq C_{1}|s|^{2} \int_{0}^{T}\|\vec{h}\|^{2} d \tau .
$$

From the last two results and (2.18) we obtain the estimate

$$
\int_{0}^{T}\|\widetilde{u}(t)\|_{1}^{2} d t \leq C_{4}|s|^{4}
$$

from which our claim follows. From the regularity of $\vec{h}$ it follows that $\widetilde{w} \in L^{\infty}((0, T)$; $W(\Omega)) \cap L^{2}((0, T) ; V(\Omega))$.

For a variation $\vec{h} \in L^{2}\left((0, T) ; L^{2}(\Omega)\right)$, the Gâteaux derivative in the direction $\vec{h}$ of the solution of the Navier-Stokes system (2.6) has been denoted by $\widetilde{w}$ and we now know it satisfies (2.13). The latter system can be written in the form

$$
\left\{\begin{array}{l}
\widetilde{w}_{t}+\nu \nabla^{2} \widetilde{w}+(\widetilde{w} \cdot \nabla) \vec{u}+(\vec{u} \cdot \nabla) \widetilde{w}+\nabla \widetilde{q}=\vec{h}, \\
\widetilde{w} \in V(\Omega), \\
\widetilde{w}(t, \vec{x})=0, \quad \vec{x} \in \Gamma, \\
\widetilde{w}(0, \vec{x})=0, \quad \vec{x} \in \Omega .
\end{array}\right.
$$

The Gâteaux derivative gives useful information about the sensitivity of the system at a particular point $\vec{u}$ in a particular direction $\vec{h}$, but complete information requires 
one to solve (2.19) for every possible direction $\vec{h}$. Fortunately, in order to minimize the functional we need only an integral over all these directions which can more easily be obtained through the solution of a single adjoint equation. To see this, we will need the following result.

Lemma 2.4. Given $\vec{u}_{0} \in V(\Omega)$ and $\vec{U} \in \mathcal{U}_{a d}$. Let $\vec{h}$ be given in $L^{2}\left((0, T) ; L^{2}(\Omega)\right)$ and let $\widetilde{w}(\vec{h})$ be defined through (2.13). For every $\vec{h}_{2} \in L^{2}\left((0, T) ; L^{2}(\Omega)\right)$, we have

$$
\int_{\Omega}[\widehat{w} \widetilde{w}]_{0}^{T} d \vec{x}+\int_{0}^{T} \int_{\Omega} \vec{h}_{2} \widetilde{w}(\vec{h}) d \vec{x} d t=\int_{0}^{T} \int_{\Omega} \widehat{w}\left(\vec{h}_{2}\right) \vec{h} d \vec{x} d t
$$

where $\widehat{w}$ is the solution of the adjoint problem

$$
\left\{\begin{array}{l}
-\widehat{w}_{t}+\nu A \widehat{w}+C^{* *}(\vec{u}(\vec{g})) \widehat{w}=\vec{h}_{2}, \\
\widehat{w} \in V(\Omega) \\
\widehat{w}=0 \quad \forall \vec{w} \in \Gamma \\
\widehat{w}(T, \vec{x})=\vec{w}_{T}(\vec{x})
\end{array}\right.
$$

Proof. The integral on the right-hand side contains $\vec{h}$ which can be evaluated by (2.13); then we can proceed by integration by parts. We note that the integration by parts is fully justified by the regularity properties of the quantities involved. In the following, we also use the fact that the operator $A$ is self-adjoint (see [4]) and the adjoint operator of $C^{\prime}\left(\vec{u}_{g}\right)$ is $C^{\prime *}\left(\vec{u}_{g}\right)$ so that

$$
\begin{aligned}
\int_{0}^{T} & \int_{\Omega} \widehat{w}\left(\vec{h}_{2}\right) \vec{h} d \vec{x} d t=\int_{0}^{T} \int_{\Omega} \widehat{w}\left(\widetilde{w}_{t}+\nu A \widetilde{w}+C^{\prime}\left(\vec{u}_{\vec{g}}\right) \widetilde{w}\right) d \vec{x} d t \\
\quad= & \int_{0}^{T} \int_{\Omega} \widetilde{w}\left(-\widehat{w}_{t}+\nu A \widehat{w}+C^{* *}\left(\vec{u}_{\vec{g}}\right) \widehat{w}\right) d \vec{x} d t+\left.\int_{\Omega}(\widehat{w} \widetilde{w})\right|_{0} ^{T} d \vec{x} \\
& =\int_{0}^{T} \int_{\Omega} \widehat{h}_{2} \widetilde{w}(\vec{h}) d \vec{x} d t+\left.\int_{\Omega}(\widehat{w} \widetilde{w})\right|_{0} ^{T} d \vec{x} .
\end{aligned}
$$

It is easy to show that the Gâteaux derivative of the functional $\mathcal{J}(\vec{u}(\vec{g}), \vec{g})$, defined in (2.8), should vanish at the optimal solution and that the optimal control $\vec{g}$ must be proportional to the solution of a particular adjoint system.

THEOREM 2.5. Given $\vec{u}_{0} \in V(\Omega)$ and $\vec{U} \in \mathcal{U}_{a d}$. If $(\vec{u}, \vec{g})$ is an optimal pair, then we have

$$
\vec{g}=-\frac{1}{\beta} \vec{w},
$$

where $\vec{w}$ is the solution of the particular adjoint problem

$$
\left\{\begin{array}{l}
-\vec{w}_{t}+\nu \nabla^{2} \vec{w}+(\nabla \vec{u})^{T} \vec{w}-(\vec{u} \cdot \nabla) \vec{w}+\nabla q=\alpha(\vec{u}-\vec{U}), \\
\vec{w} \in V(\Omega), \\
\vec{w}(t, \vec{x})=0, \quad \vec{x} \in \Gamma, \\
\vec{w}(T, \vec{x})=\gamma(\vec{u}(T)-\vec{U}(T)), \quad \vec{x} \in \Omega,
\end{array}\right.
$$

and $\vec{g} \in L^{\infty}((0, T) ; W(\Omega)) \cap L^{2}((0, T) ; V(\Omega))$. 
Proof. Let $(\vec{u}, \vec{g})$ be an optimal pair. We compute the Gâteaux derivative of the functional $\mathcal{J}(\vec{u}(\vec{g}), \vec{g})$ in the direction of $\vec{h}$, where $\vec{u}(\vec{g})$ is the solution of $(2.6)$. Then, Lemma 2.4 completes the proof. We have

$$
\begin{aligned}
\frac{D \mathcal{J}(\vec{u}(\vec{g}), \vec{g})}{D \vec{g}} \cdot \vec{h}=\int_{0}^{T} & \int_{\Omega}\left(\alpha(\vec{u}-\vec{U})\left(\frac{D \vec{u}}{D \vec{g}} \cdot \vec{h}\right)+\beta \vec{g} \cdot \vec{h}\right) d \vec{x} d t \\
& \quad+\int_{\Omega} \gamma(\vec{u}(T, \vec{x})-\vec{U}(T, \vec{x}))\left(\frac{D \vec{u}}{D \vec{g}} \cdot \vec{h}\right)(T, \vec{x}) d \vec{x} \\
= & \int_{0}^{T} \int_{\Omega}(\alpha(\vec{u}-\vec{U}) \widetilde{w}+\beta \vec{g} \cdot \vec{h}) d \vec{x} d t+\gamma \int_{\Omega}(\vec{u}(T)-\vec{U}(T)) \widetilde{w}(T) d \vec{x} \\
= & \int_{0}^{T} \int_{\Omega}(\vec{w}+\beta \vec{g}) \cdot \vec{h} d \vec{x} d t,
\end{aligned}
$$

where $\vec{w}$ is the solution of the adjoint system (2.20). Now, from the definition of the optimal control problem, if $(\vec{u}, \vec{g})$ is an optimal solution and the Gâteaux derivative of the functional exists, the latter must be zero for all directions $\vec{h} \in L^{2}\left((0, T) ; L^{2}(\Omega)\right)$. Thus,

$$
\int_{0}^{T} \int_{\Omega}(\vec{w}+\beta \vec{g}) \cdot \vec{h} d \vec{x} d t=0 \quad \forall \vec{h} \in L^{2}\left((0, T) ; L^{2}(\Omega)\right) .
$$

By the completeness of the Hilbert space, we obtain (2.21). The regularity of $\vec{g}$ follows from the regularity properties of $\vec{w}$.

2.4. The optimality system. Thus, in order to obtain the solution of our optimal control problem we have to solve, for $\vec{u} \in L^{2}\left((0, T) ; H_{0}^{1}(\Omega)\right), p \in L^{2}\left((0, T) ; L_{0}^{2}(\Omega)\right)$, $\vec{w} \in L^{2}\left((0, T) ; H_{0}^{1}(\Omega)\right)$, and $r \in L^{2}\left((0, T) ; L_{0}^{2}(\Omega)\right)$, the Navier-Stokes system

$$
\left\{\begin{aligned}
\left\langle\vec{u}_{t}, \vec{v}\right\rangle+\nu a(\vec{u}, \vec{v})+c(\vec{u} ; \vec{u}, \vec{v})+b(\vec{v}, p)=(\vec{g}, \vec{v}) & \forall \vec{v} \in H_{0}^{1}(\Omega), \\
b(\vec{u}, q)=0 & \forall q \in L_{0}^{2}(\Omega),
\end{aligned}\right.
$$

with initial velocity $\vec{u}(0, \vec{x})=\vec{u}_{0}(\vec{x})$, the adjoint system

$$
\left\{\begin{array}{rr}
-\left\langle\vec{w}_{t}, \vec{v}\right\rangle+\nu a(\vec{w}, \vec{v})+c(\vec{w} ; \vec{u}, \vec{v})+c(\vec{u} ; \vec{w}, \vec{v})+b(\vec{v}, r) \\
=\alpha(\vec{u}-\vec{U}) & \forall \vec{v} \in H_{0}^{1}(\Omega), \\
b(\vec{w}, q)=0 & \forall q \in L_{0}^{2}(\Omega),
\end{array}\right.
$$

with final condition $\vec{w}(T, \vec{x})=\gamma(\vec{u}(T)-\vec{U}(T))$ and

$$
\vec{w}=-\beta \vec{g} .
$$

The above systems of equations are a weak formulation of the system

$$
\left\{\begin{array}{r}
\vec{u}_{t}-\nu \nabla^{2} \vec{u}+(\vec{u} \cdot \vec{\nabla}) \vec{u}+\vec{\nabla} p=\vec{g} \\
\vec{\nabla} \cdot \vec{u}=0 \\
-\vec{w}_{t}+\nu \nabla^{2} \vec{w}+(\nabla \vec{u})^{T} \vec{w}-(\vec{u} \cdot \nabla) \vec{w}+\vec{\nabla} r=\alpha(\vec{u}-\vec{U}) \\
\vec{\nabla} \cdot \vec{w}=0 \\
\vec{g}+\beta \vec{w}=0
\end{array}\right.
$$


with the same corresponding final and initial conditions and with homogeneous boundary conditions.

We note that $\vec{g}$ and $\vec{u}$ belong to $L^{\infty}((0, T) ; W(\Omega)) \cap L^{2}((0, T) ; V(\Omega))$ for all finite positive values of $\alpha, \beta$, and $\gamma$.

\section{Semidiscrete-in-time approximations.}

3.1. Semidiscretization in time of the optimality system. In order to compute the solution discussed in the previous section we need to discretize this problem in time and in space. In this section, we deal with the time discretization.

Let $\sigma_{N}=\left\{t_{n}\right\}_{n=0}^{N}$ be a partition of $[0, T]$ into equal intervals $\Delta t=T / N$ with $t_{0}=0$ and $t_{N}=T$. For each fixed $\Delta t$ (or $N$ ) and for every quantity $q(t, \vec{x})$ we associate the corresponding set $\left\{q^{(n)}(\vec{x})\right\}_{n=0}^{N}$ and a continuous piecewise linear function $q^{N}=$ $q^{N}(t, \vec{x})$ such that $q^{N}\left(t_{n}, \vec{x}\right)=q^{(n)}(\vec{x})$ for all $n=0,1, \ldots, N$. We will denote with bold letters $\mathbf{q}$ the vector $\left(q^{(1)}, q^{(2)}, \ldots, q^{(N)}\right)$ of the discrete time components. Also, the space $X^{N}$ will be denoted as $\mathbf{X}$. On this partition we define the discrete target velocity as $\vec{U}^{(n)}(\vec{x})=\vec{U}\left(t_{n}, \vec{x}\right)$ for $n=0,1, \ldots, N$ whenever $\vec{U} \in \mathcal{U}_{a d}$. The state variables $\vec{u}^{(n)} \in H_{0}^{1}(\Omega)$ and $p^{(n)} \in L_{0}^{2}(\Omega)$ are constrained to satisfy the semidiscrete Navier-Stokes equations

$$
\begin{aligned}
& \frac{1}{\Delta t}\left(\vec{u}^{(n)}-\vec{u}^{(n-1)}\right)+\nu A \vec{u}^{(n)}+C\left(\vec{u}^{(n)}\right) \vec{u}^{(n)}+B^{*} p^{(n)}=\vec{g}^{(n)}, \\
& B \vec{u}^{(n)}=0, \\
& \vec{u}^{(n)}(\vec{x})=0, \quad \vec{x} \in \Gamma,
\end{aligned}
$$

for $n=1,2, \ldots, N$, with initial velocity $\vec{u}^{(0)}=\vec{u}_{0}(\vec{x}) \in V(\Omega)$. This represents a backward Euler time discretization. Optimality is achieved by means of the minimization of the discrete functional

$$
\begin{aligned}
\mathcal{J}^{N}(\mathbf{u}, \mathbf{g})=\frac{\alpha \Delta t}{2} \sum_{n=1}^{N}\left\|\vec{u}^{(n)}-\vec{U}^{(n)}\right\|^{2} & \\
& +\frac{\beta \Delta t}{2} \sum_{n=1}^{N}\left\|\vec{g}^{(n)}\right\|^{2}+\frac{\gamma}{2}\left\|\vec{u}^{(N)}-\vec{U}^{(N)}\right\|^{2} .
\end{aligned}
$$

Of course, if $\Delta t$ tends to zero, this functional tends to the corresponding continuous functional (2.8). The semidiscrete-in-time approximate optimal control problem is then given by

$$
\begin{aligned}
& \text { given } \Delta t=T / N, \vec{u}_{0} \in V(\Omega) \text {, and } \vec{U} \in \mathcal{U}_{\text {ad }} \text {, find }(\overrightarrow{\mathbf{u}}, \mathbf{p}, \overrightarrow{\mathbf{g}}) \text { in } \mathbf{H}_{0}^{1}(\Omega) \times \\
& \mathbf{L}_{0}^{2}(\Omega) \times \mathbf{L}^{2}(\Omega) \text { such that }(\overrightarrow{\mathbf{u}}, \mathbf{p}, \overrightarrow{\mathbf{g}}) \text { is the solution of }(3.1) \text { and the cost } \\
& \text { functional (3.2) is minimized. }
\end{aligned}
$$

We note that in this formulation the value of $\vec{g}^{(0)}$ is not involved and can be arbitrarily chosen as an extension of the corresponding continuous linear function $\vec{g}^{N}(t, \vec{x})$ in $C\left((0, T) ; L^{2}(\Omega)\right)$.

3.2. Existence and consistency for the semidiscrete optimal control problem. If $\overrightarrow{\mathbf{g}}$ belongs to $\mathbf{L}^{2}(\Omega)$, then the existence of solutions of the semidiscretein-time optimal control problem can be proved. This fact is an easy consequence of the definition of the optimal control problem and the boundedness of the functional.

Lemma 3.1. Let $\Delta t=T / N, \vec{u}_{0} \in V(\Omega)$, and $\vec{U} \in \mathcal{U}_{a d}$. If $(\overrightarrow{\mathbf{u}}, \overrightarrow{\mathbf{g}})$ denotes a solution of the semidiscrete optimal control problem, then there exists a constant $C$ 
independent of $\Delta t$ such that

$$
\sum_{n=1}^{N}\left\|\vec{g}^{(n)}\right\|^{2} \Delta t \leq C \quad \text { and } \quad \sum_{n=1}^{N}\left\|\vec{u}^{(n)}\right\|^{2} \Delta t \leq C .
$$

Hence we have that $\vec{g}^{N} \in L^{2}\left((0, T) ; L^{2}(\Omega)\right)$ and $\vec{u}^{N} \in L^{2}((0, T) ; W(\Omega))$ for all $N$.

Proof. If $(\overrightarrow{\mathbf{u}}, \overrightarrow{\mathbf{g}})$ is a solution of the semidiscrete-in-time optimal control problem, then $\mathcal{J}^{N}(\overrightarrow{\mathbf{u}}(\overrightarrow{\mathbf{g}}), \overrightarrow{\mathbf{g}}) \leq \mathcal{J}^{N}(\overrightarrow{\mathbf{u}}(\mathbf{0}), \mathbf{0})$. From this inequality we have

$$
\frac{\alpha \Delta t}{2} \sum_{n=1}^{N}\left\|\vec{u}^{(n)}-\vec{U}^{(n)}\right\|^{2}+\frac{\beta \Delta t}{2} \sum_{n=1}^{N}\left\|\vec{g}^{(n)}\right\|^{2}+\frac{\gamma}{2}\left\|\vec{u}^{(N)}-\vec{U}^{N}\right\|^{2} \leq \mathcal{J}^{N}(\overrightarrow{\mathbf{u}}(\mathbf{0}), \mathbf{0}) \leq C_{1}
$$

so that the first bound in (3.3) immediately follows. Also, since $\sum_{n=1}^{N} \| \vec{u}^{(n)}-$ $\vec{U}^{(n)} \|^{2} \Delta t \leq C^{\prime}$, the second bound in (3.3) follows from the triangle inequality.

Now we can state and prove the existence of solutions of the semidiscrete-in-time optimal control problem in an open bounded domain $\Omega$ with Lipschitz-continuous boundary $\Gamma$.

TheOREM 3.2. Given $\Delta t=T / N, \vec{u}_{0} \in V(\Omega)$, and $\vec{U} \in \mathcal{U}_{a d}$. Then, there exists $(\overrightarrow{\mathbf{u}}, \mathbf{p}, \overrightarrow{\mathbf{g}})$ in $\mathbf{V}(\Omega) \times \mathbf{L}_{0}^{2}(\Omega) \times \mathbf{L}^{2}(\Omega)$ such that $(\overrightarrow{\mathbf{u}}, \mathbf{p}, \overrightarrow{\mathbf{g}})$ is the solution of (3.1) and the cost functional (3.2) is minimized.

Proof. Given $N$, let $\left\{\overrightarrow{\mathbf{g}}_{k}\right\}_{k=1}^{\infty}$ be a minimizing sequence in $\mathbf{L}^{2}(\Omega)$. Using the well-known theorem concerning semidiscrete-in-time approximations of the NavierStokes equations (see [14]), we find that the corresponding sequence $\overrightarrow{\mathbf{u}}_{k}$ is uniformly bounded in $\mathbf{V}(\Omega)$. Now, we can extract a weakly convergent subsequence and show that this subsequence converges to the solution of the optimal control problem in the semidiscrete approximation. We can write

$$
\begin{aligned}
& \vec{g}_{k}^{(n)} \rightarrow \vec{g}^{(n)} \quad \text { in } \quad W(\Omega) \quad \text { weakly, } \\
& \vec{u}_{k}^{(n)} \rightarrow \vec{u}^{(n)} \quad \text { in } \quad V(\Omega) \quad \text { weakly }
\end{aligned}
$$

for $n=1,2, \ldots, N$. By using the fact that the injection of $V(\Omega)$ into $L^{2}(\Omega)$ is compact, the second subsequence converges strongly. The lower semicontinuity of the functional in (3.2) allows the pair $(\overrightarrow{\mathbf{u}}, \overrightarrow{\mathbf{g}})$ to minimize the functional. Since we can pass to the limit in the linear and the nonlinear terms, there exists $p^{(n)} \in L^{2}(\Omega)$ such that $\left(\vec{u}^{(n)}, p^{(n)}, \vec{g}^{(n)}\right)$ also satisfies the Navier-Stokes system (3.1). In fact, since $\overrightarrow{\mathbf{u}}_{k}$ converges to $\overrightarrow{\mathbf{u}}$ strongly in $\mathbf{L}^{2}(\Omega)$, then, for any $\overrightarrow{\mathbf{z}} \in[\mathcal{V}(\Omega)]^{N}$, we have

$$
\lim _{k \rightarrow \infty} c\left(\overrightarrow{\mathbf{u}}_{k} ; \overrightarrow{\mathbf{u}}_{k}, \overrightarrow{\mathbf{z}}\right)=c(\overrightarrow{\mathbf{u}} ; \overrightarrow{\mathbf{u}}, \overrightarrow{\mathbf{z}}) .
$$

Since $[\mathcal{V}(\Omega)]^{N}$ is dense in $\mathbf{V}(\Omega)$, this is still true for any $\overrightarrow{\mathbf{w}}$ in $\mathbf{V}(\Omega)$ by a continuity argument. This allows us to pass to the limit in the semidiscrete equation and complete the proof.

We can prove the convergence of our semidiscrete optimal control problem.

Theorem 3.3. Given $\Delta t=T / N, \vec{U} \in \mathcal{U}_{a d}$, and $\vec{u}_{0} \in V(\Omega)$. For $\Delta t \rightarrow 0$ $(N \rightarrow \infty)$ the solution $\left\{\left(\vec{u}^{(n)}, \vec{g}^{(n)}\right)\right\}_{n=1}^{N}$ of the semidiscrete-in-time optimal control problem tends to the solution $(\vec{u}, \vec{g})$ of the corresponding continuous optimal control problem.

Proof. Let $\vec{u}^{\prime(n)}=\left(\vec{u}^{(n)}-\vec{u}^{(n-1)}\right) / \Delta t$, and let $\vec{u}^{\prime N}$ denote the corresponding piecewise linear function. The sequences $\left\{\vec{u}^{N}\right\}_{N=1}^{\infty},\left\{\vec{g}^{N}\right\}_{N=1}^{\infty}$, and $\left\{\vec{u}^{\prime N}\right\}_{N=1}^{\infty}$ are 
uniformly bounded in $L^{2}((0, T) ; V(\Omega)) \cap L^{\infty}((0, T) ; W(\Omega)), L^{2}\left((0, T) ; L^{2}(\Omega)\right)$, and $L^{2}\left((0, T) ; V^{*}(\Omega)\right)$, respectively. This follows from Lemma 3.1 and from the wellknown theorems for semidiscrete approximation of the Navier-Stokes equations (see Lemmas III.4.4, and III.4.6 in [14].) Hence, from these sequences we can extract subsequences such that

$$
\left\{\begin{array}{l}
\vec{u}^{N} \rightarrow \vec{u} \quad L^{2}(0, T, V(\Omega)) \quad \text { weakly } \\
\vec{u}^{N} \rightarrow \vec{u} \quad L^{\infty}(0, T, H(\Omega)) \quad \text { weak-* } \\
\vec{g}^{N} \rightarrow \vec{g} \quad L^{2}\left(0, T, L^{2}(\Omega)\right) \quad \text { weakly } \\
\frac{d \vec{u}^{(N)}}{d t} \rightarrow \vec{u}^{\prime} \quad L^{2}\left(0, T, V^{*}(\Omega)\right) \quad \text { weakly }
\end{array}\right.
$$

Let $\mathcal{H}\left((0, T) ; n, m ; V, V^{*}\right)=\left\{\vec{v} \in L^{n}((0, T) ; V): \vec{v}^{\prime} \in L^{m}\left((0, T) ; V^{*}\right)\right\}$. Since $V(\Omega) \subset W(\Omega) \subset V^{*}(\Omega)$, where $V$ and $V^{*}$ are reflexive and the injections are continuous and $V(\Omega) \subset W(\Omega)$ is compact from the Sobolev imbedding theorem, then the injection from $\mathcal{H}\left((0, T) ; 2,2, V, V^{*}\right)$ into $L^{2}((0, T) ; W)$ is compact. Hence, if a sequence $\vec{u}^{(k)}$ converges weakly in $L^{2}((0, T) ; V(\Omega))$ and $\vec{u}^{\prime(n)}$ in $L^{2}\left((0, T) ; V^{*}(\Omega)\right)$, then $\vec{u}^{(k)}$ converges strongly in $L^{2}((0, T) ; W(\Omega))$. A proof of this theorem can be found in [14]. As a consequence of this compactness theorem, the convergence of the sequence $\left\{\vec{u}^{N}\right\}_{N=1}^{\infty}$ is strong in $L^{2}((0, T) ; W(\Omega))$. Now we can pass to the limit in the system of equations and in the functional. The linear terms do not give problems. Using the fact that the sequence converges weakly in $L^{2}((0, T) ; V)$ and strongly in $L^{2}((0, T) ; W)$, we can pass to the limit in the nonlinear term; see (2.9). Thus, for $N \rightarrow \infty$, the solution of the semidiscrete optimal control problem solves the continuous optimal control problem.

3.3. First-order necessary conditions. In this subsection we derive the firstorder necessary conditions. We use a different approach to that used for the continuous problem.

Let $\mathbf{B}_{1}$ and $\mathbf{B}_{2}$ denote the following sets:

$$
\begin{aligned}
& \mathbf{B}_{1}=\mathbf{H}_{0}^{1}(\Omega) \times \mathbf{L}_{0}^{2}(\Omega) \times \mathbf{L}^{2}(\Omega), \\
& \mathbf{B}_{2}=\mathbf{H}^{-1}(\Omega) \times \mathbf{L}_{0}^{2}(\Omega) .
\end{aligned}
$$

We define the nonlinear mappings $M: \mathbf{B}_{1} \rightarrow \mathbf{B}_{2}$ as $M(\overrightarrow{\mathbf{u}}, \mathbf{p}, \overrightarrow{\mathbf{g}})=(\overrightarrow{\mathbf{f}}, \mathbf{z})$ if and only if

$$
\left\{\begin{array}{l}
\frac{1}{\Delta t}\left(\vec{u}^{(n)}-\vec{u}^{(n-1)}, \vec{v}\right)+\nu a\left(\vec{u}^{(n)}, \vec{v}\right)+c\left(\vec{u}^{(n)}, \vec{u}^{(n)}, \vec{v}\right) \\
\quad+b\left(\vec{v}, p^{(n)}\right)=\left(\vec{g}^{(n)}+\vec{f}^{(n)}, \vec{v}\right) \quad \forall \vec{v} \in H_{0}^{1}(\Omega) \quad \text { for } n=1,2, \ldots, N, \\
b\left(\vec{u}^{(n)}, q\right)=\left(z^{(n)}, q\right) \quad \forall q \in L_{0}^{2}(\Omega) \quad \text { for } n=1,2, \ldots, N, \\
\vec{u}^{(n)}=0 \quad \text { on } \Gamma \quad \text { for } n=1,2, \ldots, N, \\
\vec{u}^{(0)}=\vec{u}_{0}(\vec{x}) \in V(\Omega)
\end{array}\right.
$$

and $N: \mathbf{B}_{1} \rightarrow \mathbb{R} \times \mathbf{B}_{2}$ as $N(\overrightarrow{\mathbf{u}}, \mathbf{p}, \overrightarrow{\mathbf{g}})=(a, \overrightarrow{\mathbf{f}}, \mathbf{z})$ if and only if

$$
\left(\begin{array}{c}
\mathcal{J}^{N}(\overrightarrow{\mathbf{g}}, \overrightarrow{\mathbf{u}})-\mathcal{J}^{N}(\widehat{\mathbf{g}}, \widehat{\mathbf{u}}) \\
M(\overrightarrow{\mathbf{u}}, \mathbf{p}, \overrightarrow{\mathbf{g}})
\end{array}\right)=\left(\begin{array}{c}
a \\
(\overrightarrow{\mathbf{f}}, \mathbf{z}, \overrightarrow{\mathbf{b}})
\end{array}\right)
$$


with $\widehat{\mathbf{g}} \in \mathbf{L}^{2}(\Omega)$ and $\widehat{\mathbf{u}} \in \mathbf{H}_{0}^{1}(\Omega)$. Thus, the constraint in the semidiscrete-in-time optimal control problem can be expressed as $M(\overrightarrow{\mathbf{u}}, \mathbf{p}, \overrightarrow{\mathbf{g}})=(\overrightarrow{\mathbf{0}}, \mathbf{0})$ and the optimal control problem itself can be reformulated as

find $(\hat{\mathbf{u}}, \hat{\mathbf{p}}, \hat{\mathbf{g}})$ and $a \leq 0$ such that the equation $N(\overrightarrow{\mathbf{u}}, \mathbf{p}, \overrightarrow{\mathbf{g}})=(a, \overrightarrow{\mathbf{0}}, \mathbf{0})$ is satisfied $\forall \overrightarrow{\mathbf{g}} \in \mathbf{L}^{2}(\Omega)$.

Since we are looking for minimum points, it is natural to define the directional derivatives $M^{\prime}(\overrightarrow{\mathbf{u}}, \mathbf{p}, \overrightarrow{\mathbf{g}})$ and $N^{\prime}(\overrightarrow{\mathbf{u}}, \mathbf{p}, \hat{\mathbf{g}})$ of these nonlinear operators. Given $(\overrightarrow{\mathbf{u}}, \mathbf{p}, \overrightarrow{\mathbf{g}})$, we define the linear operators $M^{\prime}(\overrightarrow{\mathbf{u}}, \mathbf{p}, \overrightarrow{\mathbf{g}}): \mathbf{B}_{1} \rightarrow \mathbf{B}_{2}$ as $M^{\prime}(\overrightarrow{\mathbf{u}}, \mathbf{p}, \overrightarrow{\mathbf{g}}) \cdot(\widetilde{\mathbf{w}}, \widetilde{\mathbf{r}}, \widetilde{\mathbf{h}})=(\overline{\mathbf{f}}, \overline{\mathbf{z}})$ if and only if

$$
\left\{\begin{array}{r}
\frac{1}{\Delta t}\left(\widetilde{w}^{(n)}-\widetilde{w}^{(n-1)}, \vec{v}\right)+\nu a\left(\widetilde{w}^{(n)}, \vec{v}\right)+c\left(\widetilde{w}^{(n)}, \vec{u}^{(n)}, \vec{v}\right) \\
+c\left(\vec{u}^{(n)}, \widetilde{w}^{(n)}, \vec{v}\right)+b\left(\vec{v}, \widetilde{r}^{(n)}\right)=\left(\bar{f}^{(n)}+\widetilde{h}^{(n)}, \vec{v}\right) \\
\forall \vec{v} \in H_{0}^{1}(\Omega) \quad \text { for } n=1,2, \ldots, N, \\
b\left(\widetilde{w}^{(n)}, q\right)=\left(\bar{z}^{(n)}, q\right) \quad \forall q \in L_{0}^{2}(\Omega) \quad \text { for } n=1,2, \ldots, N, \\
\widetilde{w}^{(n)}=\overrightarrow{0} \quad \text { on } \Gamma \quad \text { for } n=1,2, \ldots, N, \\
\widetilde{w}^{(0)}=\overrightarrow{0}
\end{array}\right.
$$

and $N^{\prime}(\overrightarrow{\mathbf{u}}, \mathbf{p}, \overrightarrow{\mathbf{g}}): \mathbf{B}_{1} \rightarrow \mathbb{R} \times \mathbf{B}_{2}$ as $N^{\prime}(\overrightarrow{\mathbf{u}}, \mathbf{p}, \overrightarrow{\mathbf{g}}) \cdot(\widetilde{\mathbf{w}}, \widetilde{\mathbf{r}}, \widetilde{\mathbf{h}})=(\bar{a}, \overline{\mathbf{f}}, \overline{\mathbf{z}})$ if and only if

$$
\left(\begin{array}{c}
\left.\left.\left(\mathcal{J}^{N}\right)^{\prime}(\overrightarrow{\mathbf{u}}, \overrightarrow{\mathbf{g}})\right) \cdot(\widetilde{\mathbf{w}}, \widetilde{\mathbf{r}}, \widetilde{\mathbf{h}})\right) \\
\left.M^{\prime}(\overrightarrow{\mathbf{u}}, \mathbf{p}, \overrightarrow{\mathbf{g}}) \cdot(\widetilde{\mathbf{w}}, \widetilde{\mathbf{r}}, \widetilde{\mathbf{h}})\right)
\end{array}\right)=\left(\begin{array}{c}
\bar{a} \\
(\overline{\mathbf{f}}, \overline{\mathbf{z}})
\end{array}\right) .
$$

Now we have to prove that these operators are well-defined and relate them to the minimum of the functional.

Lemma 3.4. Given $\Delta t=T / N, \vec{u}_{0} \in V(\Omega)$, and $\overrightarrow{\mathbf{u}} \in \mathbf{H}_{0}^{1}(\Omega)$. Then, we have

(i) the operator $M^{\prime}(\overrightarrow{\mathbf{u}}, \mathbf{p}, \overrightarrow{\mathbf{g}})$ is onto $\mathbf{B}_{2}$;

(ii) the operator $N^{\prime}(\overrightarrow{\mathbf{u}}, \mathbf{p}, \overrightarrow{\mathbf{g}})$ has closed range in $\mathbb{R} \times \mathbf{B}_{2}$.

Proof. We set

$$
\left\{\begin{array}{l}
\nu \widetilde{a}\left(\widetilde{w}^{(n)}, \vec{v}\right)=\nu a\left(\widetilde{w}^{(n)}, \vec{v}\right)+\frac{1}{\Delta t}\left(\widetilde{w}^{(n)}, \vec{v}\right) \quad \forall \vec{v} \in H_{0}^{1}(\Omega) \quad \text { for } n=1,2, \ldots, N, \\
\left(\widetilde{f}^{(n)}, \vec{v}\right)=\left(\bar{f}^{(n)}, \vec{v}\right)+\frac{1}{\Delta t}\left(\widetilde{w}^{(n-1)}, \vec{v}\right) \quad \forall \vec{v} \in H_{0}^{1}(\Omega) \quad \text { for } n=1,2, \ldots, N .
\end{array}\right.
$$

Then, (3.7) can be rewritten as

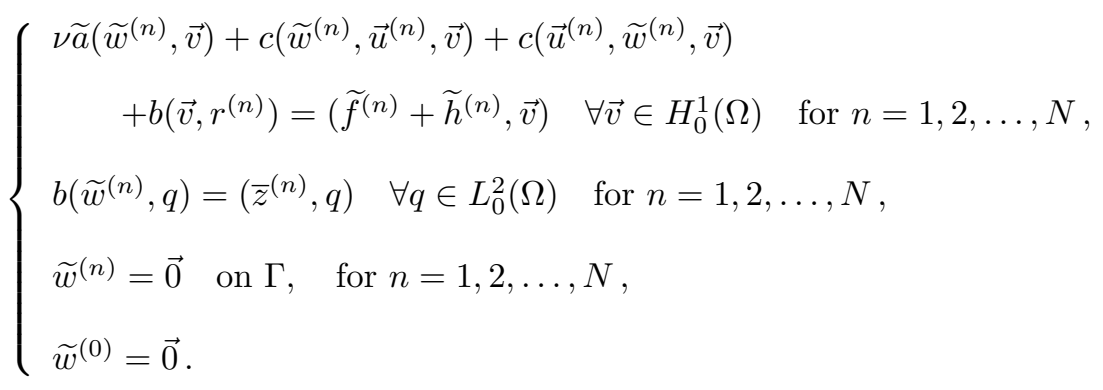


Note that since $\widetilde{f}^{(n)} \in H^{-1}(\Omega)$ whenever $\bar{f}^{(n)} \in H^{-1}(\Omega)$, the result (i) follows if it can be shown that (3.9) has a solution $(\widetilde{\mathbf{w}}, \widetilde{\mathbf{r}}, \widetilde{\mathbf{h}}) \in \mathbf{B}_{1} \forall(\widetilde{\mathbf{f}}, \overline{\mathbf{z}}) \in \mathbf{B}_{2}$. Since (3.9) is a steady-state system, one can prove this by applying the methods of [10] and [11]. However, we note that it is clear that the operator $M^{\prime}(\overrightarrow{\mathbf{u}}, \mathbf{p}, \overrightarrow{\mathbf{g}})$ is onto because, for every $\widetilde{\mathbf{w}} \in \mathbf{H}_{0}^{1}(\Omega)$ and $\overrightarrow{\mathbf{u}} \in \mathbf{H}^{3 / 2}(\Omega) \cap \mathbf{H}_{0}^{1}(\Omega)$, we can set, in $(3.9), \widetilde{\mathbf{h}}=\widetilde{\mathbf{w}} \cdot \nabla \overrightarrow{\mathbf{u}} \in \mathbf{L}^{2}(\Omega)$. Now, the existence of a solution $\widetilde{\mathbf{w}} \in \mathbf{H}_{0}^{1}(\Omega)$ for the resulting system can be proved $\forall \widetilde{\mathbf{f}} \in \mathbf{H}^{-1}(\Omega)$ and $\overline{\mathbf{z}} \in \mathbf{L}_{0}^{2}(\Omega)$ by applying standard methods.

For (ii), we note that the operator $M^{\prime}(\overrightarrow{\mathbf{u}}, \mathbf{p}, \overrightarrow{\mathbf{g}})$ belongs to $\mathcal{L}\left(\mathbf{B}_{1}, \mathbf{B}_{2}\right)$ and therefore its kernel is a closed subspace. We recall that a linear functional $f$ on a Banach space can have either the range of $f$ equals $\{0\}$ or $\{\mathbb{R}\}$. Thus, $N^{\prime}(\overrightarrow{\mathbf{u}}, \mathbf{p}, \overrightarrow{\mathbf{g}})$ acting on the kernel of $M^{\prime}(\overrightarrow{\mathbf{u}}, \mathbf{p}, \overrightarrow{\mathbf{g}})$ is either identically zero or onto $\mathbb{R}$. Let $X, Y, Z$ be Banach spaces and $A: X \rightarrow Y$ and $B: X \rightarrow Z$ be linear continuous operators. If the range of $B$ is closed in $Z$ and the subspace $A \cdot \operatorname{Ker}(B)$ is closed in $Y$, then, if we define $C: X \rightarrow Y \times Z$ by $C x=(A x, B x)$, the range of $C$ is closed in $Y \times Z$. Applying this result we have that the range of $N^{\prime}(\overrightarrow{\mathbf{u}}, \mathbf{p}, \overrightarrow{\mathbf{g}})$ is a closed set.

If $(\widehat{\mathbf{u}}, \widehat{\mathbf{p}}, \widehat{\mathbf{g}})$ denotes an optimal solution, the operator $N^{\prime}(\widehat{\mathbf{u}}, \widehat{\mathbf{p}}, \widehat{\mathbf{g}})$ cannot be onto $\mathbb{R} \times \mathbf{B}_{2}$; this in turn implies a first-order condition. (In fact, $N^{\prime}(\overrightarrow{\mathbf{u}}, \mathbf{p}, \overrightarrow{\mathbf{g}})$ cannot be onto for any $(\overrightarrow{\mathbf{u}}, \mathbf{p}, \overrightarrow{\mathbf{g}}) \in \mathbf{B}_{1}$, but we do not need so general a result here.) In the next theorem, we prove this and write out the first-order necessary condition. In the following theorem, $\langle\mathbf{f}, \mathbf{g}\rangle=\sum_{i=1}^{N}\left\langle f^{(n)}, g^{(n)}\right\rangle$ denotes a duality pairing for functions defined with respect the discrete time grid.

TheOREM 3.5. Given $\Delta t=T / N$ and $\vec{u}_{0} \in V(\Omega)$. If $(\widehat{\mathbf{u}}, \widehat{\mathbf{p}}, \widehat{\mathbf{g}}) \in\left(\mathbf{H}_{0}^{1}(\Omega) \times \mathbf{L}_{0}^{2}(\Omega) \times\right.$ $\left.\mathbf{L}^{2}(\Omega)\right)$ is a solution of the semidiscrete-in-time optimal control problem, then we have

(i) the operator $N^{\prime}(\widehat{\mathbf{u}}, \widehat{\mathbf{p}}, \widehat{\mathbf{g}})$ is not onto $\mathbb{R} \times \mathbf{B}_{2}$;

(ii) there exists a nonzero Lagrange multiplier $(\overrightarrow{\mathbf{w}}, \mathbf{r}) \in \mathbf{H}_{0}^{1}(\Omega) \times \mathbf{L}_{0}^{2}(\Omega)$ satisfying the Euler equations

$$
\begin{array}{r}
\left(\mathcal{J}^{N}\right)^{\prime}(\widehat{\mathbf{u}}, \widehat{\mathbf{g}}) \cdot(\widetilde{\mathbf{w}}, \widetilde{\mathbf{r}}, \widetilde{\mathbf{h}})-\left\langle(\overrightarrow{\mathbf{w}}, \mathbf{r}), M^{\prime}(\widehat{\mathbf{u}}, \widehat{\mathbf{p}}, \widehat{\mathbf{g}}) \cdot(\widetilde{\mathbf{w}}, \widetilde{\mathbf{r}}, \widetilde{\mathbf{h}})\right\rangle=0 \\
\forall(\widetilde{\mathbf{w}}, \widetilde{\mathbf{r}}, \widetilde{\mathbf{h}}) \in \mathbf{H}_{0}^{1}(\Omega) \times \mathbf{L}_{0}^{2}(\Omega) \times \mathbf{L}^{2}(\Omega),
\end{array}
$$

where $\langle\cdot, \cdot\rangle$ denotes the duality pairing between $\mathbf{B}_{2}$ and $\mathbf{B}_{2}^{*}$.

Proof. The operator $N^{\prime}(\widehat{\mathbf{u}}, \widehat{\mathbf{p}}, \widehat{\mathbf{g}})$ cannot be onto. If it were, by the implicit function theorem, we would have that there exists a solution, which is different from the optimal solution, that minimizes the functional for every small neighborhood of $(\widehat{\mathbf{u}}, \widehat{\mathbf{p}}, \widehat{\mathbf{g}})$. This contradicts the hypothesis that $(\widehat{\mathbf{u}}, \widehat{\mathbf{p}}, \widehat{\mathbf{g}})$ is an optimal solution.

For (ii), we note that from Lemma 3.4, the range of $N^{\prime}(\widehat{\mathbf{u}}, \widehat{\mathbf{p}}, \widehat{\mathbf{g}})$ is a closed set and from part (i) this range is a closed proper subspace of $\mathbb{R} \times \mathbf{B}_{2}$. Then, the Hahn-Banach theorem implies that there exists a nonzero element of $\mathbb{R} \times \mathbf{B}_{2}^{*}=\mathbb{R} \times \mathbf{H}_{0}^{1}(\Omega) \times \mathbf{L}_{0}^{2}(\Omega)$ that annihilates the range of $N^{\prime}(\widehat{\mathbf{u}}, \widehat{\mathbf{p}}, \widehat{\mathbf{g}})$, i.e., one can find a $(a, \overrightarrow{\mathbf{w}}, \mathbf{r}) \in \mathbb{R} \times \mathbf{B}_{2}^{*}$ such that

$$
\langle(\bar{a}, \overline{\mathbf{f}}, \overline{\mathbf{z}}),(a, \overrightarrow{\mathbf{w}}, \mathbf{r})\rangle=0 \quad \forall(\bar{a}, \overline{\mathbf{f}}, \overline{\mathbf{z}}) \in \operatorname{Ran}\left(N^{\prime}(\widehat{\mathbf{u}}, \widehat{\mathbf{p}}, \widehat{\mathbf{g}})\right),
$$

where $a \neq 0$ since this solution is nontrivial $\left(M^{\prime}(\widehat{\mathbf{u}}, \widehat{\mathbf{p}}, \widehat{\mathbf{g}})\right.$ is onto). (In $(3.11),\langle\cdot, \cdot \cdot\rangle$ denotes the duality pairing between $\mathbb{R} \times \mathbf{B}_{2}$ and $\mathbb{R} \times \mathbf{B}_{2}^{*}$ and $\operatorname{Ran}\left(N^{\prime}(\widehat{\mathbf{u}}, \widehat{\mathbf{p}}, \widehat{\mathbf{g}})\right)$ the range of $N^{\prime}(\widehat{\mathbf{u}}, \widehat{\mathbf{p}}, \widehat{\mathbf{g}})$.) We may, in fact, set $\bar{a}=-1$ and then (3.10) follows from (3.11) and the definition $(3.8)$ of $N^{\prime}(\overrightarrow{\mathbf{u}}, \mathbf{p}, \overrightarrow{\mathbf{g}})$.

3.4. The optimality system. We can rewrite the first-order necessary condition (3.10) as a system of partial differential equations. Solutions of this system are also solutions of the semidiscrete-in-time optimal control problem. 
Theorem 3.6. Given $\Delta t=T / N$ and $\vec{u}_{0} \in V(\Omega)$. Let $(\overrightarrow{\mathbf{u}}, \mathbf{p}, \overrightarrow{\mathbf{g}}) \in \mathbf{H}_{0}^{1}(\Omega) \times \mathbf{L}_{0}^{2}(\Omega) \times$ $\mathbf{L}^{2}(\Omega)$ ) denote a solution of the semidiscrete-in-time optimal control problem. Then, we have

$$
\beta \vec{g}^{(n)}=-\vec{w}^{(n-1)} \quad \text { for } n=1,2, \ldots, N
$$

where $(\overrightarrow{\mathbf{w}}, \mathbf{r}) \in \mathbf{H}_{0}^{1}(\Omega) \times \mathbf{L}_{0}^{2}(\Omega)$ satisfies

$$
\left\{\begin{array}{c}
-\frac{1}{\Delta t}\left(\vec{w}^{(n)}-\vec{w}^{(n-1)}, \vec{v}\right)+\nu a\left(\vec{v}, \vec{w}^{(n-1)}\right)+c\left(\vec{v}, \vec{u}^{(n)}, \vec{w}^{(n-1)}\right) \\
+c\left(\vec{u}^{(n)}, \vec{v}, \vec{w}^{(n-1)}\right)+b\left(\vec{v}, r^{(n-1)}\right)=\alpha\left(\vec{u}^{(n)}-\vec{U}^{(n)}, \vec{v}\right) \\
\forall \vec{v} \in H_{0}^{1}(\Omega) \quad \text { for } n=1, \ldots, N, \\
b\left(\vec{w}^{(n-1)}, q\right)=0 \quad \forall q \in L_{0}^{2}(\Omega) \quad \text { for } n=1, \ldots, N, \\
\vec{w}^{(n-1)}=\overrightarrow{0} \quad \text { on } \Gamma \quad \text { for } n=1, \ldots, N, \\
\vec{w}^{(N)}=\gamma\left(\vec{u}^{(N)}-\vec{U}^{(N)}\right) .
\end{array}\right.
$$

Proof. From (3.2) and (3.7), the first-order necessary condition (3.10) is equivalent to

$$
\begin{gathered}
\alpha \sum_{n=1}^{N}\left(\left(\vec{u}^{(n)}-\vec{U}^{(n)}\right), \widetilde{w}^{(n)}\right) \Delta t+\gamma\left(\left(\vec{u}^{(N)}-\vec{U}^{(N)}\right), \widetilde{w}^{(N)}\right)+\beta \sum_{n=1}^{N}\left(\vec{g}^{(n)}, \widetilde{h}^{(n)}\right) \Delta t \\
-\sum_{n=1}^{N}\left[\frac{1}{\Delta t}\left(\widetilde{w}^{(n)}-\widetilde{w}^{(n-1)}, \vec{w}^{(n-1)}\right)+\nu a\left(\widetilde{w}^{(n)}, \vec{w}^{(n-1)}\right)+c\left(\widetilde{w}^{(n)}, \vec{u}^{(n)}, \vec{w}^{(n-1)}\right)\right. \\
\left.+c\left(\vec{u}^{(n)}, \widetilde{w}^{(n)}, \vec{w}^{(n-1)}\right)+b\left(\vec{w}^{(n-1)}, \widetilde{r}^{(n)}\right)-\left(\widetilde{h}^{(n)}, \vec{w}^{(n-1)}\right)+b\left(\widetilde{w}^{(n)}, r^{(n-1)}\right)\right] \Delta t=0
\end{gathered}
$$

$\forall(\widetilde{\mathbf{w}}, \widetilde{\mathbf{r}}, \widetilde{\mathbf{h}}) \in \mathbf{B}_{1}=\mathbf{H}_{0}^{1}(\Omega) \times \mathbf{L}_{0}^{2}(\Omega) \times \mathbf{L}^{2}(\Omega)$. One easily sees, using $\widetilde{w}^{(0)}=0$ (see $(3.7))$, that

$$
\sum_{n=1}^{N}\left(\widetilde{w}^{(n)}-\widetilde{w}^{(n-1)}, \vec{w}^{(n-1)}\right)=-\sum_{i=1}^{N}\left(\widetilde{w}^{(n)}, \vec{w}^{(n)}-\vec{w}^{(n-1)}\right)+\left(\widetilde{w}^{(N)}, \vec{w}^{(N)}\right) .
$$

Combining the above equations and choosing $\widetilde{w}^{(n)}=0$ and $\widetilde{r}^{(n)}=0 \forall n$, one easily finds that

$$
\sum_{n=1}^{N} \int_{\Omega}\left(\beta \vec{g}^{(n)}+\vec{w}^{(n-1)}, \widetilde{h}^{(n)}\right) d \vec{x}
$$

As the variations $\widetilde{h}^{(n)}$ are independent in $\mathbf{L}^{2}(\Omega)$, we obtain (3.12).

Choosing $\widetilde{w}^{(n)}=0$ and $\widetilde{h}^{(n)}=0$ leads, in a similar manner, to $b\left(\vec{w}^{(n)}, q\right)=0$ for all $q \in L_{0}^{2}(\Omega)$. 
Finally, choosing $\widetilde{r}^{(n)}=0$ and $\widetilde{h}^{(n)}=0$ yields

$$
\begin{aligned}
& \left(\gamma\left(\vec{u}^{(N)}-\vec{U}^{(N)}\right)-\vec{w}^{(N)}, \widetilde{w}^{(N)}\right) \\
& -\Delta t \sum_{n=1}^{N}\left[-\frac{1}{\Delta t}\left(\widetilde{w}^{(n)}, \vec{w}^{(n)}-\vec{w}^{(n-1)}\right)+\nu a\left(\widetilde{w}^{(n)}, \vec{w}^{(n-1)}\right)\right. \\
& +c\left(\widetilde{w}^{(n)}, \vec{u}^{(n)}, \vec{w}^{(n-1)}\right)+c\left(\vec{u}^{(n)}, \widetilde{w}^{(n)}, \vec{w}^{(n-1)}\right) \\
& \left.\quad+b\left(\widetilde{w}^{(n)}, r^{(n-1)}\right)-\alpha\left(\left(\vec{u}^{(n)}-\vec{U}^{(n)}\right), \widetilde{w}^{(n)}\right)\right]=0
\end{aligned}
$$

from which the remaining equations in (3.13) follow.

Thus, in order to solve the semidiscrete-in-time optimal control problem, we have to solve the semidiscrete Navier-Stokes system (3.1), which can be expressed as

$$
\left\{\begin{array}{l}
\frac{1}{\Delta t}\left(\vec{u}^{(n)}-\vec{u}^{(n-1)}, \vec{v}\right)+\nu a\left(\vec{u}^{(n)}, \vec{v}\right)+c\left(\vec{u}^{(n)} ; \vec{u}^{(n)}, \vec{v}\right) \\
\quad+b\left(\vec{v}, p^{(n)}\right)=\left(\vec{g}^{(n)}, \vec{v}\right) \quad \forall \vec{v} \in H_{0}^{1}(\Omega), \\
b\left(\vec{u}^{(n)}, q\right)=0 \quad \forall q \in L_{0}^{2}(\Omega) \\
\vec{u}^{(n)}=0 \quad \text { on } \Gamma \\
\vec{u}^{(0)}=\vec{u}_{0} \quad \text { in } \Omega,
\end{array}\right.
$$

the semidiscrete adjoint system (3.13), and the optimality condition (3.12).

\section{Fully discrete time-space approximation.}

4.1. Preliminaries. We consider only conforming finite element approximations. Let $X^{h} \subset H_{0}^{1}(\Omega)$ and $S^{h} \subset L^{2}(\Omega)$ be two families of finite dimensional subspaces parameterized by $h$ that tends to zero. We let $S_{0}^{h}=S^{h} \cap L_{0}^{2}(\Omega)$. We make the following assumptions on $X^{h}$ and $S^{h}$.

(a) Approximation properties: there exists an integer $l$ and a constant $C$, independent of $h, \vec{u}$ and $p$, such that for $1 \leq k \leq l$ we have

$$
\begin{aligned}
& \inf _{\vec{u}_{h} \in X^{h}}\left\|\vec{u}_{h}-\vec{u}\right\|_{1} \leq C h^{k}\|\vec{u}\|_{k+1} \quad \forall \vec{u} \in H^{k+1}(\Omega) \cap H_{0}^{1}(\Omega), \\
& \inf _{p_{h} \in S^{h}}\left\|p-p_{h}\right\| \leq C h^{k}\|p\|_{k} \quad \forall p \in H^{k}(\Omega) \cap L_{0}^{2}(\Omega) .
\end{aligned}
$$

(b) The inf-sup condition or $L B B$ condition: there exists a constant $C^{\prime}$, independent of $h$ such that

$$
\inf _{0 \neq q_{h} \in S^{h}} \sup _{0 \neq \vec{u}_{h} \in X^{h}} \frac{b\left(\vec{u}_{h}, q_{h}\right)}{\left\|\vec{u}_{h}\right\|_{1}\left\|q_{h}\right\|} \geq C^{\prime}>0 .
$$

The condition (4.3) ensures the stability of solutions of the discretized Navier-Stokes system.

In order to preserve the antisymmetry of the trilinear form $c(\vec{u} ; \vec{v}, \vec{w})$ on the finite element spaces, we introduce the modified trilinear form (see [14])

$$
\widetilde{c}(\vec{u} ; \vec{v}, \vec{w})=\frac{1}{2}\{c(\vec{u} ; \vec{v}, \vec{w})-c(\vec{u} ; \vec{w}, \vec{v})\} \quad \forall \vec{u}, \vec{v}, \vec{w} \in H^{1}(\Omega) .
$$


We can recall some useful formulas and inequalities that hold in a two-dimensional domain $\Omega$ :

$$
\begin{gathered}
c(\vec{u} ; \vec{v}, \vec{w})=\widetilde{c}(\vec{u} ; \vec{v}, \vec{w}) \quad \forall \vec{u} \in H_{0}^{1}(\Omega) \cap W(\Omega) \quad \forall \vec{v}, \vec{w} \in H_{0}^{1}(\Omega), \\
\left\{\begin{array}{l}
\widetilde{c}(\vec{u} ; \vec{v}, \vec{w})=-\widetilde{c}(\vec{u} ; \vec{w}, \vec{v}) \quad \forall \vec{u}, \vec{v}, \vec{w} \in H_{0}^{1}(\Omega), \\
\widetilde{c}(\vec{u} ; \vec{v}, \vec{v})=0 \quad \forall \vec{u}, \vec{v} \in H_{0}^{1}(\Omega)
\end{array}\right.
\end{gathered}
$$

and (see [14])

$$
\left\{\begin{array}{l}
|\widetilde{c}(\vec{u} ; \vec{v}, \vec{w})| \leq K_{1}\|\nabla \vec{u}\| \cdot\|\vec{v}\|_{L^{4}(\Omega)}\|\nabla \vec{w}\| \quad \forall \vec{u}, \vec{v}, \vec{w} \in H_{0}^{1}(\Omega), \\
|\widetilde{c}(\vec{u} ; \vec{v}, \vec{w})| \leq K_{2}\|\vec{u}\|^{\frac{1}{2}}\|\nabla \vec{u}\|^{\frac{1}{2}}\|\nabla \vec{v}\|\|\vec{w}\|^{\frac{1}{2}}\|\nabla \vec{w}\|^{\frac{1}{2}} \quad \forall \vec{u}, \vec{v}, \vec{w} \in H_{0}^{1}(\Omega) .
\end{array}\right.
$$

We remark that the second inequality in (4.5) is true in the framework of the conforming finite element approximation and only in the two-dimensional case (see [14]).

4.2. Formulation of the fully discrete optimal control approximation. Let $\sigma_{N}=\left\{t_{n}\right\}_{n=0}^{N}$ be a partition of $[0, T]$ into equal intervals $\Delta t=T / N$ with $t_{0}=0$ and $t_{N}=T$. For each fixed $\Delta t$ (or $N$ ) and for every quantity $q(t, \vec{x})$, we associate the corresponding set $\left\{q_{h}^{(n)}\right\}_{n=1}^{N}$. We will denote the vector $\left(q_{h}^{(1)}, q_{h}^{(2)}, \ldots, q_{h}^{(N)}\right)$ as $\mathbf{q}_{h}$ and the space $Y^{N}$ as $\mathbf{Y}$. We also define the continuous piecewise linear function $\vec{q}_{h}^{N}(t, \vec{x})$ by the conditions $\vec{q}_{h}^{N}\left(t_{n}, \vec{x}\right)=q_{h}\left(t_{n}, \vec{x}\right) \forall n=0,1,2, \ldots, N$.

Given $\Delta t=T / N, \overrightarrow{\mathbf{g}} \in \mathbf{L}^{2}(\Omega)$ and $\vec{u}_{0} \in V(\Omega),\left(\overrightarrow{\mathbf{u}}_{h}, \mathbf{p}_{h}\right)$ is called a generalized solution of the time-space discretization of the Navier-Stokes system if $u_{h}^{(n)} \in X^{h}$ and $p_{h}^{(n)} \in S_{0}^{h}$ and $\left(u_{h}^{(n)}, p_{h}^{(n)}\right)$ satisfy the system of equations

$$
\left\{\begin{array}{c}
\frac{1}{\Delta t}\left(\vec{u}_{h}^{(n)}-\vec{u}_{h}^{(n-1)}, \vec{v}_{h}\right)+\nu a\left(\vec{u}_{h}^{(n)}, \vec{v}_{h}\right)+\widetilde{c}\left(\vec{u}_{h}^{(n)} ; \vec{u}_{h}^{(n)}, \vec{v}_{h}\right) \\
+b\left(\vec{v}_{h}, p^{(n)}\right)=\left(\vec{g}^{(n)}, \vec{v}_{h}\right) \quad \forall \vec{v}_{h} \in X^{h}(\Omega) \\
b\left(\vec{v}_{h}^{(n)}, q_{h}\right)=0 \quad \forall q_{h} \in S_{0}^{h}(\Omega)
\end{array}\right.
$$

for $n=1,2, \ldots, N$ with initial velocity $\vec{u}_{h}^{(0)}=\pi^{h} \vec{u}_{0}(\vec{x})$.

The discrete functional used in the optimal control problem is given by

$$
\begin{aligned}
\mathcal{J}_{h}^{N}\left(\overrightarrow{\mathbf{u}}_{h}, \overrightarrow{\mathbf{g}}_{h}\right)= & \frac{\alpha \Delta t}{2} \sum_{n=1}^{N}\left\|\vec{u}_{h}^{(n)}-\vec{U}^{(n)}\right\|^{2} \\
& +\frac{\beta \Delta t}{2} \sum_{n=1}^{N}\left\|\vec{g}^{(n)}\right\|^{2}+\frac{\gamma}{2}\left\|\vec{u}_{h}^{(N)}-\vec{U}^{N}\right\|^{2} .
\end{aligned}
$$

The formulation of the fully discrete optimal control problem is given by given $\Delta t=T / N, \vec{u}_{0} \in V(\Omega)$, and $\vec{U} \in \mathcal{U}_{a d}$, find $\left(\overrightarrow{\mathbf{u}}_{h}, \mathbf{p}_{h}, \overrightarrow{\mathbf{g}}_{h}\right)$ in $\mathbf{X} h(\Omega) \times \mathbf{S}_{0}^{h}(\Omega) \times \mathbf{S}^{h}(\Omega)$ such that (4.6) is satisfied and the cost functional (4.7) is minimized. 
The existence and the convergence of solutions of the fully discrete optimal control problem can be proved in the same way as for the semidiscrete case if we limit our analysis to conforming finite elements approximations. We can state that the solution $\left(\overrightarrow{\mathbf{u}}_{h}, \overrightarrow{\mathbf{g}}_{h}\right)$ of the fully discrete optimal control problem tends to the optimal control solution $(\overrightarrow{\mathbf{u}}, \overrightarrow{\mathbf{g}})$ of the continuous problem as $\Delta t \rightarrow 0(N \rightarrow \infty)$ and $h \rightarrow 0$. The wellknown corresponding theorems for the full space-time discretization of the NavierStokes equations can be found in [14]. The necessary optimality condition can be found using the same techniques as those used in [10], which are essentially the same as were used for the semidiscrete case. For error estimates, one can refer to [10], [12], and [14]. Finally, for completeness, we can state the theorem that gives the control as a solution of an adjoint problem.

THEOREM 4.1. Let $\vec{u}_{0} \in V(\Omega), \vec{U} \in \mathcal{U}_{a d}$, and $\Delta t=T / N$. If $\left(\overrightarrow{\mathbf{u}}_{h}, \overrightarrow{\mathbf{g}}_{h}\right)$ is a solution of the fully discrete optimal control problem, then we have $\vec{g}_{h}^{(n)}=-\frac{1}{\beta} \vec{w}_{h}^{(n-1)} \forall n=$ $1,2, \ldots, N$. For $n=0,1, \ldots, N$, the functions $\vec{w}_{h}^{(n)} \in X^{h}$ are the solution of the adjoint problem

$$
\left\{\begin{array}{c}
-\frac{1}{\Delta t}\left(\vec{w}_{h}^{(n)}-\vec{w}_{h}^{(n-1)}, \vec{v}_{h}\right)+\nu a\left(\vec{v}_{h}, \vec{w}_{h}^{(n-1)}\right)+\widetilde{c}\left(\vec{v}_{h} ; \vec{u}_{h}^{(n)}, \vec{w}_{h}^{(n-1)}\right) \\
+\widetilde{c}\left(\vec{u}_{h}^{(n)} ; \vec{v}_{h}, \vec{w}_{h}^{(n-1)}\right)+b\left(\vec{v}_{h}, r_{h}^{(n-1)}\right) \\
=\alpha\left(\vec{u}_{h}^{(n)}-\vec{U}^{(n)}, \vec{v}_{h}\right) \quad \forall \vec{v}_{h} \in X^{h}(\Omega) \\
b\left(\vec{w}_{h}^{(n-1)}, q_{h}\right)=0 \quad \forall q_{h} \in S_{0}^{h}(\Omega)
\end{array}\right.
$$

for $n=1,2, \ldots, N$ along with the terminal condition $\vec{w}_{h}^{(N)}=\gamma\left(\vec{u}_{h}^{(N)}-\vec{U}^{(N)}\right)$.

5. A gradient method. We now consider a gradient method for solution of the fully discrete optimal control problem. Due to the forward-in-time nature of the state equations and the backward-in-time nature of the adjoint equations, any practical algorithm would involve a split of the optimality system into two parts. Thus, the fully discrete system consists of

(a) the Navier-Stokes system: for $n=1,2, \ldots, N, \vec{u}_{h}^{(n)} \in X^{h}, p_{h}^{(n)} \in S_{0}^{h}$, and

$$
\begin{cases}\frac{1}{\Delta t}\left(\vec{u}_{h}^{(n)}-\vec{u}_{h}^{(n-1)},\right. & \left.\vec{v}_{h}\right)+\nu a\left(\vec{u}_{h}^{(n)}, \vec{v}_{h}\right)+\widetilde{c}\left(\vec{u}_{h}^{(n)} ; \vec{u}_{h}^{(n)}, \vec{v}_{h}\right) \\ & +b\left(\vec{v}_{h}, p_{h}^{(n)}\right)=\left(\vec{g}_{h}^{(n)}, \vec{v}_{h}\right) \quad \forall \vec{v}_{h} \in X^{h}(\Omega), \\ b\left(\vec{u}_{h}^{(n)}, q_{h}\right)=0 \quad \forall q_{h} \in S_{0}^{h}(\Omega) & \end{cases}
$$

with initial velocity $\vec{u}_{h}^{(0)}=\pi^{h} \vec{u}_{0}(\vec{x})$;

(b) the adjoint system: for $n=1,2, \ldots, N, \vec{w}_{h}^{(n-1)} \in X^{h}, r_{h}^{(n-1)} \in S_{0}^{h}$, and

$$
\left\{\begin{array}{c}
-\frac{1}{\Delta t}\left(\vec{w}_{h}^{(n)}-\vec{w}_{h}^{(n-1)}, \vec{v}_{h}\right)+\nu a\left(\vec{v}_{h}, \vec{w}_{h}^{(n-1)}\right)+\widetilde{c}\left(\vec{v}_{h} ; \vec{u}_{h}^{(n)}, \vec{w}_{h}^{(n-1)}\right) \\
+\widetilde{c}\left(\vec{u}_{h}^{(n)} ; \vec{v}_{h}, \vec{w}_{h}^{(n-1)}\right)+b\left(\vec{v}_{h}, r_{h}^{(n-1)}\right) \\
=\alpha\left(\vec{u}_{h}^{(n)}-\vec{U}^{(n)}, \vec{v}_{h}\right) \quad \forall \vec{v}_{h} \in X^{h}(\Omega), \\
b\left(\vec{w}_{h}^{(n-1)}, q_{h}\right)=0 \quad \forall q_{h} \in S_{0}^{h}(\Omega)
\end{array}\right.
$$


with terminal condition $\vec{w}_{h}^{(N)}=-\gamma\left(\vec{u}_{h}^{(N)}-\vec{U}^{(N)}\right)$.

The optimal control variable $\vec{g}_{h}^{(n)}$ is related to the adjoint velocity $\vec{w}_{h}^{(n)}$ by $\vec{g}_{h}^{(n)}=$ $\frac{1}{\beta} \vec{w}_{h}^{(n-1)}$. In the gradient algorithm, we invoke this relation only after convergence is achieved.

Let $\mathcal{J}_{h}^{N}(k)=\mathcal{J}_{h}^{N}\left(\overrightarrow{\mathbf{u}}_{h}(k), \overrightarrow{\mathbf{g}}_{h}(k)\right)$, where $\mathcal{J}_{h}^{N}(\cdot, \cdot)$ is given by (4.7) and $k$ is the iteration counter of the gradient algorithm. In the algorithm, $\tau$ will denote a prescribed tolerance used to test for the convergence of the functional. The gradient algorithm proceeds as follows.

(a) initialization:

(i) choose $\tau$ and $\overrightarrow{\mathbf{g}}_{h}(0)$; set $k=0$ and $\epsilon=1$;

(ii) solve for the starting velocity field $\overrightarrow{\mathbf{u}}_{h}(0)$ from (5.1) with $\overrightarrow{\mathbf{g}}_{h}=\overrightarrow{\mathbf{g}}_{h}(0)$;

(b) main loop:

(iii) evaluate $\mathcal{J}_{h}^{N}(0)$;

(iv) set $k=k+1$;

(v) solve for $\overrightarrow{\mathbf{w}}_{h}(k)$ from (5.2) with $\overrightarrow{\mathbf{u}}_{h}=\overrightarrow{\mathbf{u}}_{h}(k-1)$;

(vi) set $\overrightarrow{\mathbf{g}}_{h}(k)=\overrightarrow{\mathbf{g}}_{h}(k-1)-\epsilon\left(\beta \overrightarrow{\mathbf{g}}_{h}(k-1)-\overrightarrow{\mathbf{w}}_{h}(k)\right)$;

(vii) solve for $\overrightarrow{\mathbf{u}}_{h}(k)$ from (5.1) with $\overrightarrow{\mathbf{g}}_{h}=\overrightarrow{\mathbf{g}}_{h}(k)$;

(viii) evaluate $\mathcal{J}_{h}^{N}(k)$;

(ix) if $\mathcal{J}_{h}^{N}(k) \geq \mathcal{J}_{h}^{N}(k-1)$, set $\epsilon=.5 \epsilon$ and go to (vi); otherwise, continue;

(x) if $\left|\mathcal{J}_{h}^{N}(k)-\mathcal{J}_{h}^{N}(k-1)\right| /\left|\mathcal{J}_{h}^{N}(k)\right|>\tau$, set $\epsilon=1.5 \epsilon$ and go to (iv); otherwise, stop.

The bulk of the computational costs are found in the backward-in-time solution of the discrete adjoint system in step (v) and the forward-in-time solution of the discrete state system in step (vii).

The convergence property of the gradient algorithm is given in the following result.

TheOREm 5.1. Let $\left(\overrightarrow{\mathbf{u}}_{h}(k), \overrightarrow{\mathbf{w}}_{h}(k), \mathbf{p}_{h}(k), \mathbf{r}_{h}(k), \overrightarrow{\mathbf{g}}_{h}(k)\right)$ denote the $k$ th iterate of the gradient algorithm and let $\left(\overrightarrow{\mathbf{u}}_{h}, \overrightarrow{\mathbf{w}}_{h}, \mathbf{p}_{h}, \mathbf{r}_{h}, \overrightarrow{\mathbf{g}}_{h}\right)$ denote the solution of the fully discrete optimality system (5.1) and (5.2). Then, for $\Delta t$ sufficiently small there exists a ball $B$ in $L^{2}\left((0, T) ; X^{h}\right)$ whose radius depends on $\alpha, \beta$, and $\gamma$, such that if $\overrightarrow{\mathbf{g}}_{h}(0) \in B$, then

$$
\left(\overrightarrow{\mathbf{u}}_{h}(k), \overrightarrow{\mathbf{w}}_{h}(k), \mathbf{p}_{h}(k), \mathbf{r}_{h}(k), \overrightarrow{\mathbf{g}}_{h}(k)\right) \rightarrow\left(\overrightarrow{\mathbf{u}}_{h}, \overrightarrow{\mathbf{w}}_{h}, \mathbf{p}_{h}, \mathbf{r}_{h}, \overrightarrow{\mathbf{g}}_{h}\right) \quad \text { as } k \rightarrow \infty
$$

Proof. First, recall the following classical result; see, e.g., [3]. Let $X$ be a Hilbert space with norm $\|\cdot\|$ and inner product $\langle\cdot, \cdot\rangle$. Let $\mathcal{J}(\cdot)$ be a real-valued functional on $X$. Suppose that $\mathcal{J}(\cdot)$ is of class $C^{2}$, that it has a local minimum at a point $g \in X$, and that there exist two real numbers $c_{1}$ and $c_{2}$ and a ball $B \subset X$ centered at $g$ such that $\forall \delta g_{1}, \delta g_{2} \in X$ and $\forall \widetilde{g} \in B$ we have that

$$
\mathcal{J}^{\prime \prime}(\widetilde{g})\left(\delta g_{1}, \delta g_{2}\right) \leq c_{1}\left\|\delta g_{1}\right\|\left\|\delta g_{2}\right\| \quad \text { and } \quad\left\|\delta g_{1}\right\|^{2} c_{2} \leq \mathcal{J}^{\prime \prime}(\widetilde{g})\left(\delta g_{1}, \delta g_{1}\right),
$$

where $\mathcal{J}^{\prime \prime}(\widetilde{g})\left(\delta g_{1}, \delta g_{2}\right)$ is the bilinear form associated with the second derivatives of $\mathcal{J}(\cdot)$. Then, the gradient algorithm converges, for any $g_{0} \in B$, to $g$.

Now, for our setting, let $\Delta t=T / N$. Then, for each $\widetilde{\mathbf{g}}_{h} \in L^{2}\left((0, T) ; \mathbf{X}^{h}\right)$, the second Frechet derivative of $\mathcal{J}_{h}^{N}\left(\widetilde{\mathbf{u}}_{h}\left(\widetilde{\mathbf{g}}_{h}\right), \widetilde{\mathbf{g}}_{h}\right)$ is given by

$$
D^{2} \mathcal{J}_{h}^{N}\left(\widetilde{\mathbf{u}}_{h}\left(\widetilde{\mathbf{g}}_{h}\right), \widetilde{\mathbf{g}}_{h}\right)\left(\delta \overrightarrow{\mathbf{g}}_{1 h}, \delta \overrightarrow{\mathbf{g}}_{2 h}\right)=\alpha \Delta t \sum_{n=1}^{N} \int_{\Omega} \widetilde{w}_{1 h}^{(n)} \cdot \widetilde{w}_{2 h}^{(n)} d \vec{x}
$$




$$
\begin{aligned}
& +\beta \Delta t \sum_{n=1}^{N} \int_{\Omega} \delta \vec{g}_{1 h}^{(n)} \cdot \delta \vec{g}_{2 h}^{(n)} d \vec{x}+\gamma \int_{\Omega} \widetilde{w}_{1 h}^{(N)} \cdot \widetilde{w}_{2 h}^{(N)} d \vec{x} \\
& +\alpha \Delta t \sum_{n=1}^{N} \int_{\Omega}\left(\vec{u}_{h}^{(n)}-\vec{U}_{h}^{(n)}\right) \cdot \widetilde{z}_{h}^{(n)} d \vec{x}+\gamma \int_{\Omega}\left(\vec{u}_{h}^{(N)}-\vec{U}_{h}^{(N)}\right) \cdot \widetilde{z}_{h}^{(N)} d \vec{x},
\end{aligned}
$$

where $\widetilde{u}_{h}^{(n)} \in X^{h}$ is the solution of $\widetilde{u}_{h}^{(0)}=\pi^{h} \vec{u}_{0}(\vec{x})$ and

$$
\left\{\begin{aligned}
\frac{1}{\Delta t}\left(\widetilde{u}_{h}^{(n)}-\widetilde{u}_{h}^{(n-1)},\right. & \left.\vec{v}_{h}\right)+\nu a\left(\widetilde{u}_{h}^{(n)}, \vec{v}_{h}\right)+\widetilde{c}\left(\widetilde{u}_{h}^{(n)} ; \widetilde{u}_{h}^{(n)}, \vec{v}_{h}\right) \\
& +b\left(\vec{v}_{h}, p_{h}^{(n)}\right)=\left(\widetilde{g}_{h}^{(n)}, \vec{v}_{h}\right) \quad \forall \vec{v}_{h} \in X^{h}(\Omega) \\
b\left(\widetilde{u}_{h}^{(n)}, q_{h}\right)=0 \quad \forall q_{h} \in S_{0}^{h}(\Omega) &
\end{aligned}\right.
$$

for $n=1,2, \ldots, N$, the first variations $\widetilde{w}_{1 h}^{(n)} \in X^{h}$ and $\widetilde{w}_{2 h}^{(n)} \in X^{h}$ are solutions of $\widetilde{w}_{i h}^{(0)}=\overrightarrow{0}$ and

$$
\left\{\begin{array}{l}
\frac{1}{\Delta t}\left(\widetilde{w}_{i h}^{(n)}-\widetilde{w}_{i h}^{(n-1)}, \vec{v}_{h}\right)+\nu a\left(\widetilde{w}_{i h}^{(n)}, \vec{v}_{h}\right)+\widetilde{c}\left(\widetilde{w}_{i h}^{(n)} ; \widetilde{u}_{h}^{(n)}, \vec{v}_{h}\right) \\
+\widetilde{c}\left(\widetilde{u}_{h}^{(n)} ; \widetilde{w}_{i h}^{(n)}, \vec{v}_{h}\right)+b\left(\vec{v}_{h}, \widetilde{r}_{i h}^{(n)}\right)=\left(\delta \vec{g}_{i h}^{(n)}, \vec{v}_{h}\right) \quad \forall \vec{v}_{h} \in X^{h}(\Omega), \\
b\left(\widetilde{w}_{i h}^{(n)}, q_{h}\right)=0 \quad \forall q_{h} \in S_{0}^{h}(\Omega)
\end{array}\right.
$$

for $n=1,2, \ldots, N$ and for $i=1,2$, respectively, and the second variation $\widetilde{z}_{h}^{(n)} \in X^{h}$ is the solution of $\widetilde{z}_{h}^{(0)}=\overrightarrow{0}$ and

$$
\left\{\begin{array}{c}
\frac{1}{\Delta t}\left(\widetilde{z}_{h}^{(n)}-\widetilde{z}_{h}^{(n-1)}, \vec{v}_{h}\right)+\nu a\left(\widetilde{z}_{h}^{(n)}, \vec{v}_{h}\right)+\widetilde{c}\left(\widetilde{z}_{h}^{(n)} ; \widetilde{u}_{h}^{(n)}, \vec{v}_{h}\right)+\widetilde{c}\left(\widetilde{u}_{h}^{(n)} ; \widetilde{z}_{h}^{(n)}, \vec{v}_{h}\right) \\
+b\left(\vec{v}_{h}, \widetilde{s}_{h}^{(n)}\right)=-\widetilde{c}\left(\widetilde{w}_{1 h}^{(n)} ; \widetilde{w}_{2 h}^{(n)}, \vec{v}_{h}\right)-\widetilde{c}\left(\widetilde{w}_{2 h}^{(n)} ; \widetilde{w}_{1 h}^{(n)}, \vec{v}_{h}\right) \quad \forall \vec{v}_{h} \in X^{h}(\Omega) \\
b\left(\widetilde{z}_{h}^{(n)}, q_{h}\right)=0 \quad \forall q_{h} \in S_{0}^{h}(\Omega)
\end{array}\right.
$$

for $n=1,2, \ldots, N$. and

Let $\widetilde{w}_{h}^{(n)} \in X^{h}$ be the solution of the adjoint system $\widetilde{w}_{h}^{(N)}=-\gamma\left(\widetilde{u}_{h}^{(N)}-\vec{U}^{(N)}\right)$

$$
\left\{\begin{array}{c}
-\frac{1}{\Delta t}\left(\widetilde{w}_{h}^{(n)}-\widetilde{w}_{h}^{(n-1)}, \vec{v}_{h}\right)+\nu a\left(\vec{v}_{h}, \widetilde{w}_{h}^{(n-1)}\right)+\widetilde{c}\left(\vec{v}_{h} ; \widetilde{u}_{h}^{(n)}, \widetilde{w}_{h}^{(n-1)}\right) \\
+\widetilde{c}\left(\widetilde{u}_{h}^{(n)} ; \vec{v}_{h}, \widetilde{w}_{h}^{(n-1)}\right)+b\left(\vec{v}_{h}, r_{h}^{(n-1)}\right) \\
=\alpha\left(\widetilde{u}_{h}^{(n)}-\vec{U}^{(n)}, \vec{v}_{h}\right) \quad \forall \vec{v}_{h} \in X^{h}(\Omega) \\
b\left(\widetilde{w}_{h}^{(n-1)}, q_{h}\right)=0 \quad \forall q_{h} \in S_{0}^{h}(\Omega)
\end{array}\right.
$$

for $n=1,2, \ldots, N$. Then, combining (5.4), (5.7), and (5.8), we have that

$$
D^{2} \mathcal{J}_{h}^{N}\left(\widetilde{\mathbf{u}}_{h}\left(\widetilde{\mathbf{g}}_{h}\right), \widetilde{\mathbf{g}}_{h}\right)\left(\delta \overrightarrow{\mathbf{g}}_{1 h}, \delta \overrightarrow{\mathbf{g}}_{2 h}\right)=\alpha \Delta t \sum_{n=1}^{N}\left(\widetilde{w}_{1 h}^{(n)}, \widetilde{w}_{2 h}^{(n)}\right)
$$




$$
\begin{aligned}
& +\beta \Delta t \sum_{n=1}^{N}\left(\delta \vec{g}_{1 h}^{(n)}, \delta \vec{g}_{2 h}^{(n)}\right)+\gamma\left(\widetilde{w}_{1 h}^{(N)}, \widetilde{w}_{2 h}^{(N)}\right) \\
& -\Delta t \sum_{n=1}^{N}\left(\widetilde{c}\left(\widetilde{w}_{1 h}^{(n)} ; \widetilde{w}_{2 h}^{(n)}, \widetilde{w}_{h}^{(n-1)}\right)+\widetilde{c}\left(\widetilde{w}_{2 h}^{(n)} ; \widetilde{w}_{1 h}^{(n)}, \widetilde{w}_{h}^{(n-1)}\right)\right) .
\end{aligned}
$$

Let $\overrightarrow{\mathbf{g}}_{h}$ be the initial guess in the ball $B$ of radius $\xi$, i.e., $\left\|\overrightarrow{\mathbf{g}}_{h}-\widetilde{\mathbf{g}}_{h}\right\|=\rho \leq \xi$. Now, we have to show that there exists a $\xi$ such that $\forall \rho \leq \xi$, (5.3) is satisfied for some $c_{1}$ and $c_{2}$.

Without any restrictions on $\Delta t$ we have

$$
\left\|\widetilde{\mathbf{u}}_{h}^{(n)}\right\|_{1} \leq C\left(\left\|\widetilde{\mathbf{g}}_{h}-\overrightarrow{\mathbf{g}}_{h}\right\|+K\right) .
$$

From (5.6), if $\Delta t$ has been chosen in an appropriate way, we can obtain the estimate

$$
\left\|\widetilde{\mathbf{w}}_{i h}\right\|_{1} \leq f_{1}\left(\left\|\overrightarrow{\mathbf{g}}_{h}-\widetilde{\mathbf{g}}_{h}\right\|\right)\left\|\delta \overrightarrow{\mathbf{g}}_{i h}\right\|
$$

for $i=1,2$ and where $f_{1}(\cdot)$ is a continuous function. To see this, first note that (5.6) results in

$$
\begin{aligned}
& \left(1-2 K_{4} \Delta t\left\|\widetilde{u}_{h}^{(n)}\right\|_{1}^{2}\right)\left\|\widetilde{w}_{i h}^{(n)}\right\|^{2}-\left\|\widetilde{w}_{i h}^{(n-1)}\right\|^{2} \\
& \quad+2 \Delta t(\nu-2 \epsilon)\left\|\widetilde{w}_{i h}^{(n)}\right\|_{1}^{2} \leq 2 \Delta t K_{3}\left\|\delta \vec{g}_{i h}^{(n)}\right\|^{2}
\end{aligned}
$$

or

$$
G^{(n)}\left\|\widetilde{w}_{i h}^{(n)}\right\|^{2}-\left\|\widetilde{w}_{i h}^{(n-1)}\right\|^{2}+2 \Delta t(\nu-2 \epsilon)\left\|\widetilde{w}_{i h}^{(n)}\right\|_{1}^{2} \leq 2 \Delta t K_{3}\left\|\delta \vec{g}_{i h}^{(n)}\right\|^{2},
$$

where

$$
G^{(n)}=1-\Delta t\left(2 K_{4}\left\|\widetilde{u}_{h}^{(n)}\right\|_{1}^{2}\right) .
$$

We note that (5.12) is true for every $\Delta t$, but if

$$
\Delta t \leq \frac{1}{2 K_{4} C^{2}\left(\left\|\widetilde{\mathbf{g}}_{h}-\overrightarrow{\mathbf{g}}_{h}\right\|+K\right)^{2}},
$$

then $G^{(n)}>0$ and thus

$$
\begin{gathered}
\left(\prod_{k=1}^{n} G^{(k)}\right)\left\|\widetilde{w}_{i h}^{(n)}\right\|_{0}^{2}+2 \Delta t(\nu-2 \epsilon) \sum_{m=1}^{n}\left(\prod_{k=1}^{m-1} G^{(k)}\right)\left\|\widetilde{w}_{i h}^{(m)}\right\|_{1}^{2} \\
\leq 2 \Delta t K_{3} \sum_{m=1}^{n}\left(\prod_{k=1}^{m-1} G^{(k)}\right)\left\|\delta \vec{g}_{i h}^{(m)}\right\|^{2},
\end{gathered}
$$

where for $n=0, \prod_{k=1}^{n-1} G^{(k)} \equiv 1$. If (5.15) holds, then $G^{(n)}>0$ so that

$$
\prod_{k=1}^{m-1} G^{(k)} \leq e^{-2 \Delta t K_{4} \sum_{k=1}^{m-1}\left\|\widetilde{u}_{h}^{(k)}\right\|_{1}^{2}} \leq 1, \quad \prod_{k=1}^{n} G^{(k)}>0
$$


and

$$
\Delta t(\nu-2 \epsilon) \sum_{m=1}^{n}\left(\prod_{k=1}^{m-1} G^{(k)}\right)\left\|\widetilde{w}_{i h}^{(m)}\right\|_{1}^{2} \leq \Delta t K_{3} \sum_{m=1}^{n}\left\|\delta \vec{g}_{i h}^{(m)}\right\|^{2}=K_{3}\left\|\delta \overrightarrow{\mathbf{g}}_{i h}\right\|^{2} .
$$

Now, if $\Delta t$ satisfies the more restrictive condition

$$
\Delta t \leq \frac{1}{4 K_{4} C^{2}\left(\left\|\widetilde{\mathbf{g}}_{h}-\overrightarrow{\mathbf{g}}_{h}\right\|+K\right)^{2}},
$$

then

$$
\prod_{k=1}^{m-1} G^{(k)} \geq e^{-4 \Delta t K_{4} \sum_{k=1}^{m-1}\left\|\widetilde{u}_{h}^{(k)}\right\|_{1}^{2}} \geq e^{-4 \Delta t K_{4} \sum_{k=1}^{N}\left\|\widetilde{u}_{h}^{(k)}\right\|_{1}^{2}}=e^{-4 K_{4}\left\|\widetilde{\mathbf{u}}_{h}\right\|_{1}^{2}}
$$

so that, from (5.17),

$$
\left\|\widetilde{\mathbf{w}}_{i h}\right\|_{1}^{2} \leq\left(\frac{K_{3}}{(\nu-2 \epsilon) \Delta t} e^{4 K_{4}\left\|\widetilde{\mathbf{u}}_{h}\right\|_{1}^{2}}\right)\left\|\delta \overrightarrow{\mathbf{g}}_{i h}\right\|^{2} .
$$

Combining with (5.10), we have that (5.11) holds if (5.18) holds. Note that the time step restriction (5.18) does not involve the spatial grid size $h$.

In the same manner, from (5.8), we can obtain the estimate

$$
\left\|\overrightarrow{\mathbf{w}}_{h}\right\|_{1} \leq \alpha f_{2}\left(\left\|\overrightarrow{\mathbf{g}}_{h}-\widetilde{\mathbf{g}}_{h}\right\|\right),
$$

where $f_{2}(\cdot)$ is a continuous function.

Then, using (5.11) and (5.19) in (5.9), we have that for some constant $K>0$

$$
\begin{aligned}
& \left|D^{2} \mathcal{J}_{h}^{N}\left(\widetilde{\mathbf{u}}_{h}\left(\widetilde{\mathbf{g}}_{h}\right), \widetilde{\mathbf{g}}_{h}\right)\left(\delta \overrightarrow{\mathbf{g}}_{1 h}, \delta \overrightarrow{\mathbf{g}}_{2 h}\right)\right| \\
& \quad \leq\left(\beta+\left(\alpha+\gamma+\alpha K f_{2}\left(\left\|\overrightarrow{\mathbf{g}}_{h}-\widetilde{\mathbf{g}}_{h}\right\|\right)\right) f_{1}^{2}\left(\left\|\overrightarrow{\mathbf{g}}_{h}-\widetilde{\mathbf{g}}_{h}\right\|\right)\right)\left\|\delta \overrightarrow{\mathbf{g}}_{1 h}\right\|\left\|\delta \overrightarrow{\mathbf{g}}_{2 h}\right\|
\end{aligned}
$$

and

$$
\begin{aligned}
& \left|D^{2} \mathcal{J}_{h}^{N}\left(\widetilde{\mathbf{u}}_{h}\left(\widetilde{\mathbf{g}}_{h}\right), \widetilde{\mathbf{g}}_{h}\right)\left(\delta \overrightarrow{\mathbf{g}}_{1 h}, \delta \overrightarrow{\mathbf{g}}_{1 h}\right)\right| \\
& \quad \geq\left(\beta-\alpha K f_{2}\left(\left\|\overrightarrow{\mathbf{g}}_{h}-\widetilde{\mathbf{g}}_{h}\right\|\right) f_{1}^{2}\left(\left\|\overrightarrow{\mathbf{g}}_{h}-\widetilde{\mathbf{g}}_{h}\right\|\right)\right)\left\|\delta \overrightarrow{\mathbf{g}}_{1 h}\right\|^{2} .
\end{aligned}
$$

Now, from the continuity of $f_{1}$ and $f_{2}$ we have that for every positive $\epsilon$ there exists a $\xi>0$ such that if $\left\|\widetilde{\mathbf{g}}_{h}-\overrightarrow{\mathbf{g}}_{h}\right\| \leq \xi$ then $f_{1}\left(\left\|\overrightarrow{\mathbf{g}}_{h}-\widetilde{\mathbf{g}}_{h}\right\|\right) \leq \epsilon$ and $f_{2}\left(\left\|\overrightarrow{\mathbf{g}}_{h}-\widetilde{\mathbf{g}}_{h}\right\|\right) \leq \epsilon$. This yields

$$
\begin{aligned}
& \left|D^{2} \mathcal{J}_{h}^{N}\left(\widetilde{\mathbf{u}}_{h}\left(\widetilde{\mathbf{g}}_{h}\right), \widetilde{\mathbf{g}}_{h}\right)\left(\delta \overrightarrow{\mathbf{g}}_{1 h}, \delta \overrightarrow{\mathbf{g}}_{2 h}\right)\right| \\
& \left.\quad \leq\left(\beta+(\alpha+\gamma+\alpha K \epsilon) \epsilon^{2}\right)\right)\left\|\delta \overrightarrow{\mathbf{g}}_{1 h}\right\|\left\|\delta \overrightarrow{\mathbf{g}}_{2 h}\right\| \leq c_{2}\left\|\delta \overrightarrow{\mathbf{g}}_{1 h}\right\|\left\|\delta \overrightarrow{\mathbf{g}}_{2 h}\right\|
\end{aligned}
$$

and

$$
\left|D^{2} \mathcal{J}_{h}^{N}\left(\widetilde{\mathbf{u}}_{h}\left(\widetilde{\mathbf{g}}_{h}\right), \widetilde{\mathbf{g}}_{h}\right)\left(\delta \overrightarrow{\mathbf{g}}_{1 h}, \delta \overrightarrow{\mathbf{g}}_{1 h}\right)\right| \geq\left(\beta-\alpha K \epsilon^{3}\right)\left\|\delta \overrightarrow{\mathbf{g}}_{1 h}\right\|^{2} \geq c_{2}\left\|\delta \overrightarrow{\mathbf{g}}_{1 h}\right\|^{2},
$$

where

$$
c_{1}=\beta+(\alpha+\gamma+\alpha K \epsilon) \epsilon^{2} \quad \text { and } \quad c_{2}=\beta-\alpha K \epsilon^{3} .
$$


If $\epsilon$ is chosen such that $\beta-\alpha K \epsilon^{3} \geq 0$ then there exists a $\xi$ such that $\forall\left\|\overrightarrow{\mathbf{g}}_{h}-\widetilde{\mathbf{g}}_{h}\right\|=\rho \leq \xi$ (5.20) and (5.21) imply the inequalities in (5.3) with $\Delta t \leq \frac{1}{4 K_{4} C^{2}(\xi+K)^{2}}$.

REMARK 5.2. As noted in the proof, the restriction on the time step $\Delta t$ does not depend on the spatial grid.

REMARK 5.3. The restriction on the time step $\Delta t$ is a necessary condition and not sufficient for determining the radius of the ball. Therefore, a given $\Delta t$ cannot determine the radius of the ball which is determined by the functions $f_{1}$ and $f_{2}$ if the prescribed time step is used to solve the first variation equations.

6. Computational experiments. We consider a unit square domain $\Omega=$ $(0,1) \times(0,1) \subset \mathbb{R}^{2}$. We assume that the time interval $[0,1]$ is divided in equal intervals of time $\Delta t=1 / N$. The Taylor-Hood pair of finite element spaces is used on a rectangular mesh, i.e., the finite element spaces are chosen to be continuous piecewise biquadratic polynomials for the velocity and continuous piecewise bilinear polynomials for the pressure. The mesh size is $h$ and calculations with varying mesh sizes have been performed. All the vector plots are normalized by the maximum values.

6.1. Example 1. We are interested in the convergence history with respect to all the parameters involved so that a simple stationary target velocity $\vec{U}=(U, V)$ is chosen, where

$$
U(x, y)=10 \frac{d}{d y}(\phi(x) \phi(y)) \quad \text { and } \quad V(x, y)=-10 \frac{d}{d x}(\phi(x) \phi(y))
$$

with

$$
\phi(z)=(1-\cos (0.8 \pi z))(1-z)^{2} .
$$

Note that $\vec{U}$ is divergence free.

6.1.1. Velocity tracking evolution. We first examine an example of control for the initial velocity

$$
u_{0}(x, y)=-10 U(x, y) \quad \text { and } \quad v_{0}(x, y)=-10 V(x, y) .
$$

We set $\Delta t=0.0125, h=1 / 16, \alpha=1, \beta=0.0001$, and $\gamma=0.5$.

The evolution is given in Figures 6.1.1-6.1.1. The controlled fluid is depicted on the left and the desired flow on the right. Observe that we start with a high energy flow that rotates in the direction opposite direction to that of the target flow. Also observe that we can divide the evolution into four distinct phases:

(i) decreasing the magnitude: at the beginning the control does not act on the shape of the flow but on the magnitude;

(ii) changing the shape: at the bottom of Figure 6.1.1, we have a rapid change in shape; the change occurs so quickly that it is difficult to track it even with small time steps;

(iii) matching the target flow: the control changes shape and magnitude in order to match the target flow (at the top of Figure 6.1.1); at $t=.25$ we achieve a near perfect match;

(iv) tracking the velocity: the control keeps tracking the target flow; the effect of control in this steady phase is excellent and improves near $t=T=1$.

Figure 6.3 shows the error $\|\vec{u}-\vec{U}\|$ between the controlled flow $\vec{u}$ and the target flow $\vec{U}$ and the norm of the control $\|\vec{g}\|$. We see the error rapidly goes to zero and that 


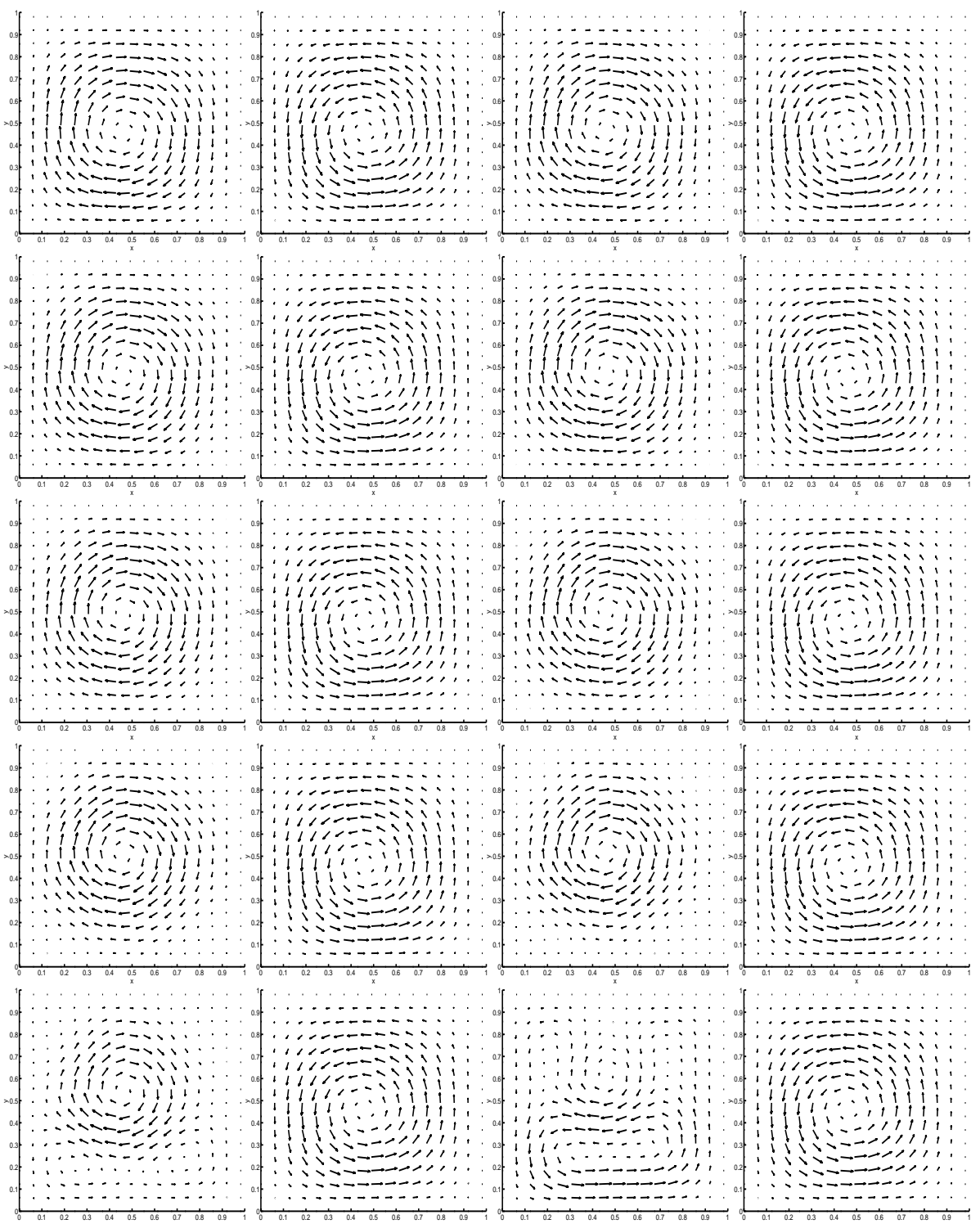

FIG. 6.1. Test 1. Controlled (first and third columns) and target (second and fourth columns) flows at $t=0$ and .05 (first row), $t=.1$ and .125 (second row), $t=.15$ and .163 (third row), $t=.169$ and .175 (fourth row), and $t=.181$ and .187 (fifth row).

the control works hard, i.e., its norm is relatively large, at the beginning in order to steer the controlled flow to the target flow and then, after a good match is achieved, its norm remains relatively constant and small. Near $t=T=1$, the control strength increases in order to minimize the term in the functional that is evaluated at $t=T$.

6.1.2. Dependence on $\boldsymbol{\beta}$ and $\gamma$. We now examine the effects of changes in the parameters $\beta$ and $\gamma$. The initial velocity is set to zero. In Figure 6.4, we show the error $\|\vec{u}-\vec{U}\|$ between the controlled flow $\vec{u}$ and the target flow $\vec{U}$ and the norm of the control $\|\vec{g}\|$ for different values of $\beta$. Starting from the top in the upper figure, 


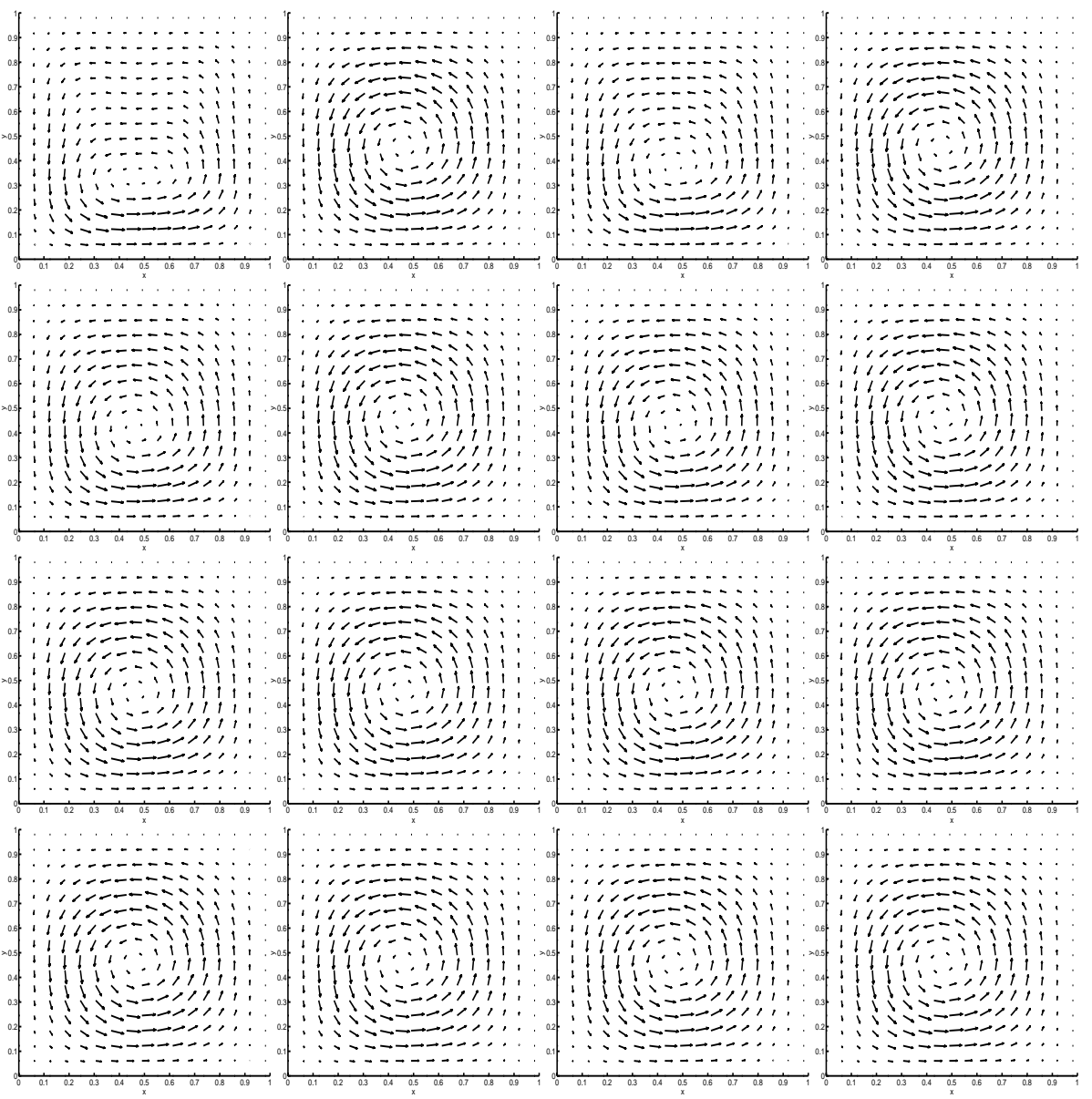

FIG. 6.2. Test 1. Controlled (first and third columns) and target (second and fourth columns) flows at $t=.193$ and .2 (first row), $t=.212$ and .225 (second row), $t=.25$ and .3 (third row), and $t=.5$ and 1 (fourth row).

we have $\beta=0.01,0.001,0.0001$, and 0.00001 . The corresponding curves in the lower figure can be identified as running from bottom to top along the vertical axis. The value of $\gamma$ for these calculations is held constant at 0.5 . The time step $\Delta t$ is again 0.0125 and $h=1 / 16$. We note that the controlled flow matches the target flow very well for values of $\beta<0.001$. The reduction of the error in the tracking phase near $t=T=1$ is accompanied by a reduction of the error in the matching phase near $t=0$ when $\beta$ decreases. The norm of the control agrees with the intuitive behavior of the error. For low values of $\beta$, the control resembles a delta function at $t=0$ plus the body force generated by the target velocity $\vec{U}$.

From the results for different values of $\gamma$, we see the importance of the term in the functional evaluated at $t=T$. In Figure 6.5 , we see the error $\|\vec{u}-\vec{U}\|$ between the controlled flow $\vec{u}$ and the target flow $\vec{U}$ for $\gamma=0.5,0.1$, and 0.01 . For small values of $\gamma$, the controlled flow tends to wander far from the target flow near $t=T$. This is not acceptable or desirable. This effect is more pronounced if $\beta$ is small. Generally, effecting good control requires small values of $\beta$; however, if $\gamma$ is also small, the error near $t=T$ could be greater than that near $t=0$ and thus the tracking performance 

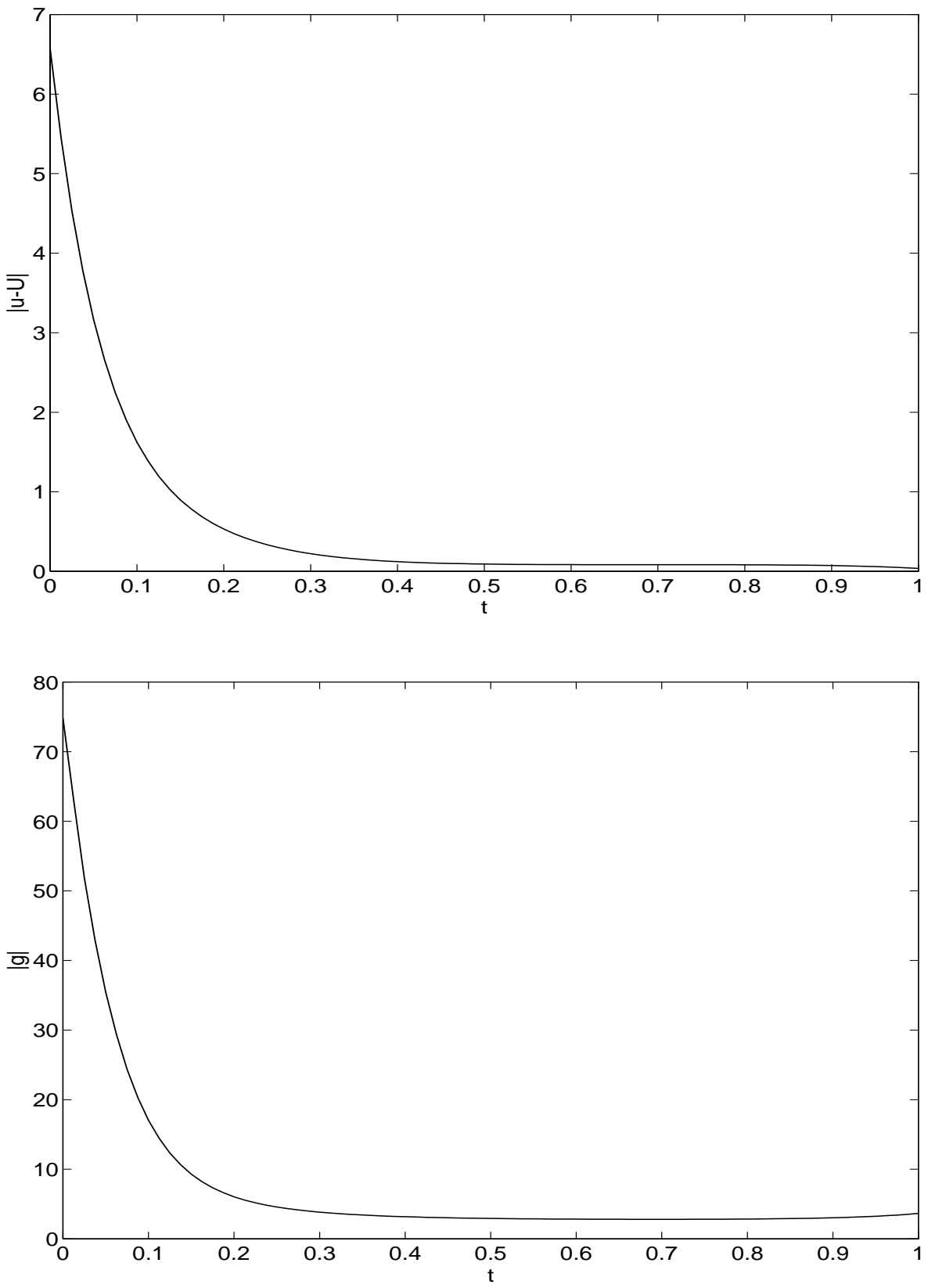

FIG. 6.3. Test 1. Error $\|\vec{u}-\vec{U}\|$ (top) and control norm $\|g\|$ (bottom).

of the control is not acceptable. As expected, the control $\vec{g}$ approaches zero at $t=T$ when $\gamma$ goes to zero so that, in that limit, the error near $t=T$ is not controllable. The conclusion that can be reached from these observations is that the term in the functional evaluated at $t=T$ is necessary for good control. A good choice for $\gamma$ is about 0.5 for most values of $\beta$. 

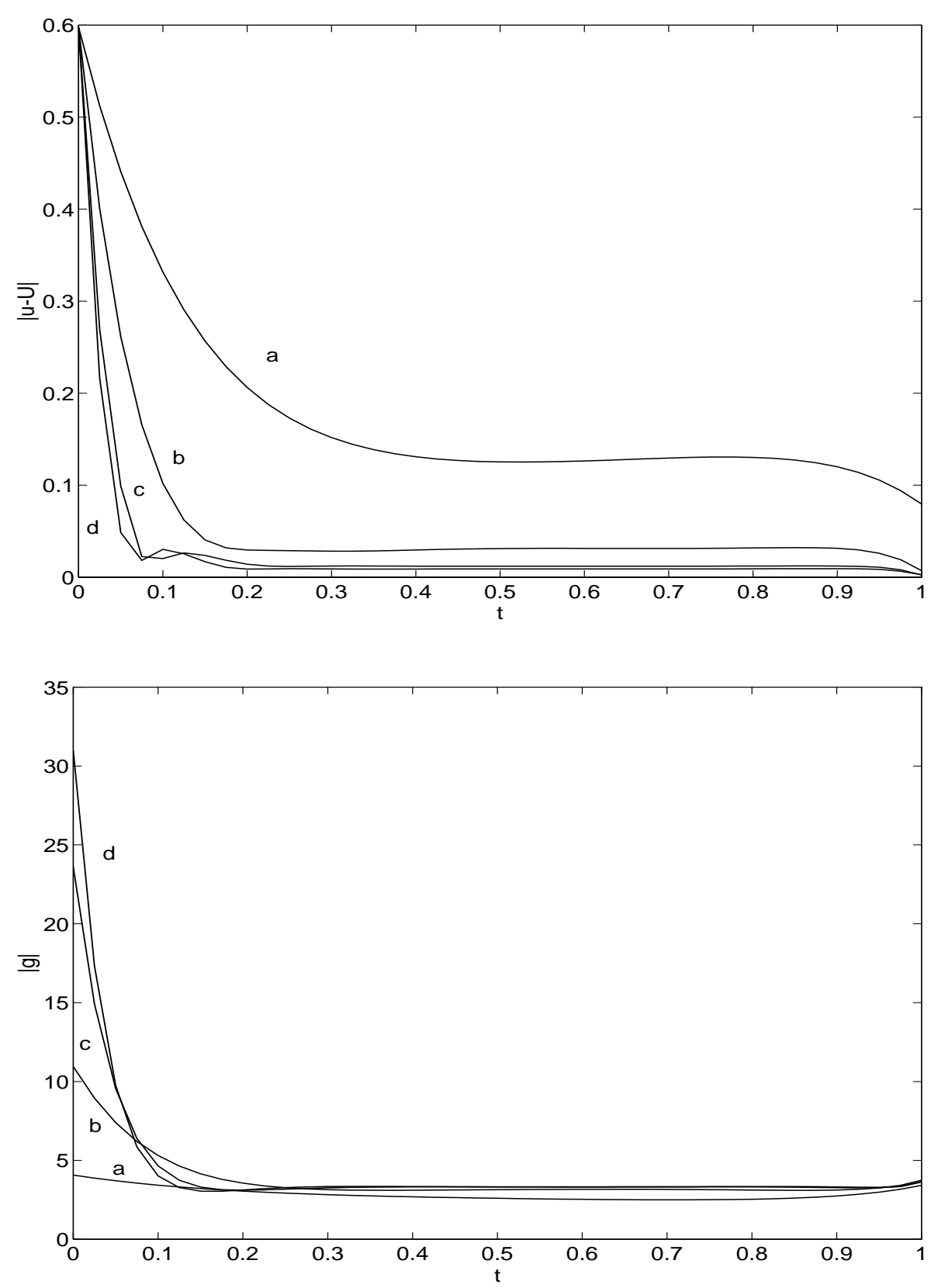

FIG. 6.4. Test 1. Error (top) and control norm (bottom) for different $\beta$.

6.1.3. Dependence on $\boldsymbol{h}$ and $\boldsymbol{T}$. In Figure 6.6 , we see the error $\|\vec{u}-\vec{U}\|$ between the controlled flow $\vec{u}$ and the target flow $\vec{U}$ for $h=1 / 8,1 / 12$, and $1 / 16$. The initial velocity has been chosen to be zero and $\beta=1 / 3000, \gamma=0.5$, and $\Delta t=0.0125$. Because of the simple form of the target velocity, good spatial accuracy is achieved in all these cases. Some differences are visible around $t=T=1$ since this point is very sensitive to the discretization. On the bottom of Figure 6.6, we have the error for different values of $T(T=1$ (a), $T=2(\mathrm{~b})$, and $T=4(\mathrm{c}))$ for $\beta=0.001$ and $\gamma=0.1$. 

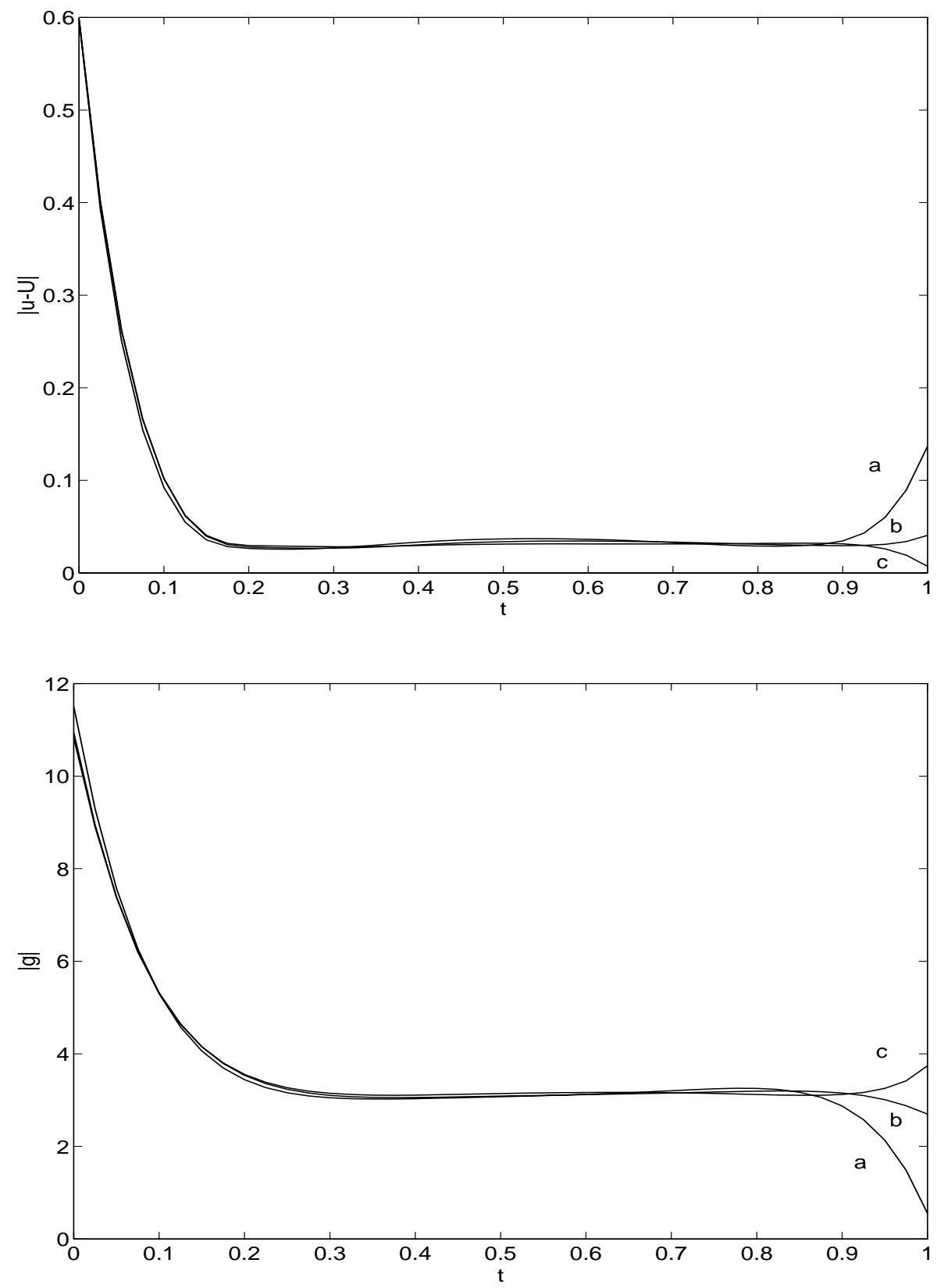

FIG. 6.5. Test 1. Error (top) and control norm (bottom) for different $\gamma$.

It seems that changes in the value of the final time $T$ does not affect the time history of the state or control in the overlapping time intervals.

6.2. Test 2. The target velocity $\vec{U}$ for this test is a solution of the Stokes system with zero initial velocity and body force $\vec{f}=\left(f_{1}, f_{2}\right)$ given by

$$
f_{1}=a(0.4, x, y)-e^{-2 t} a(0.6, x, y) \quad \text { and } \quad f_{2}=b(0.4, x, y)-e^{-2 t} b(0.6, x, y),
$$



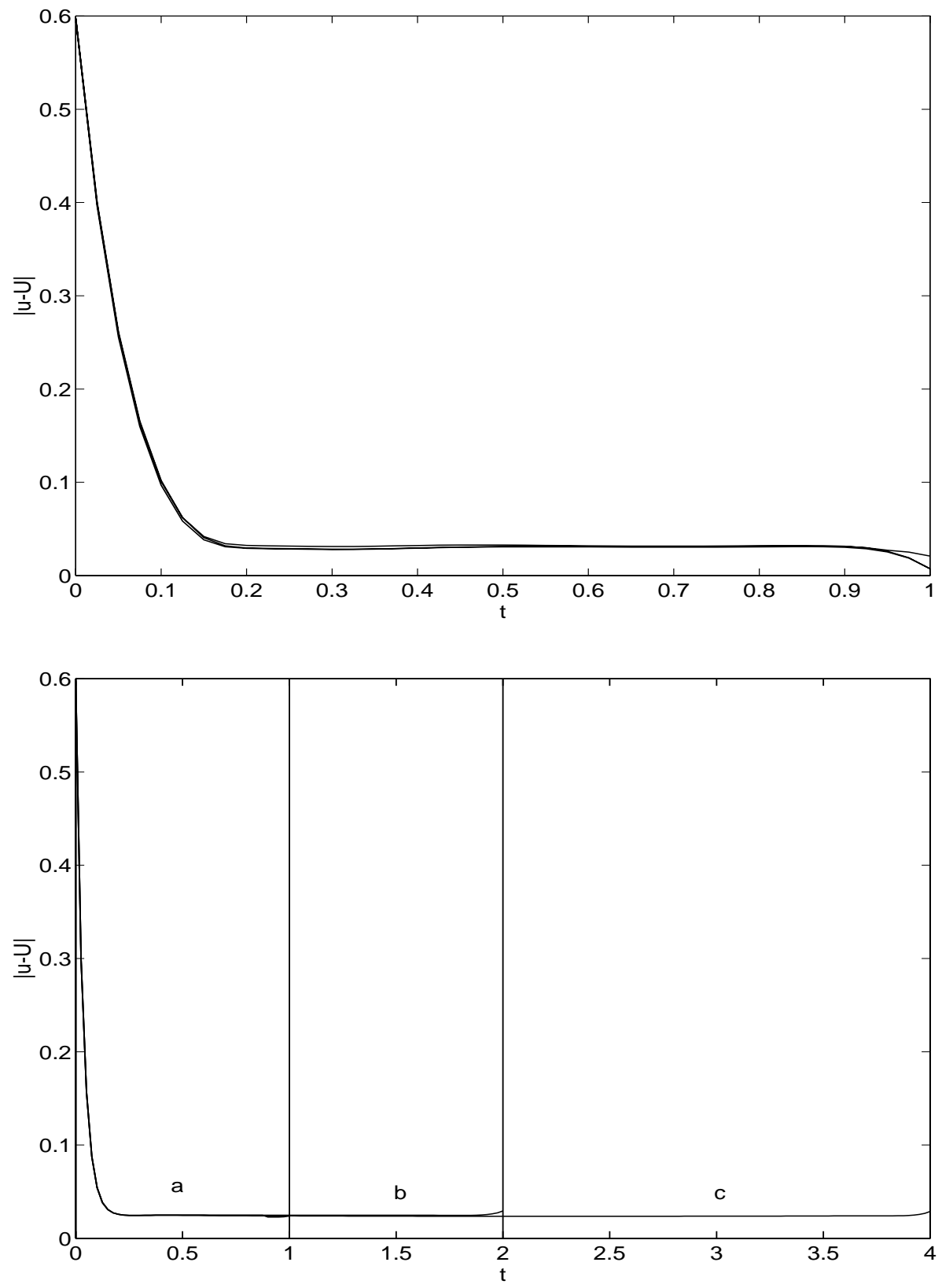

FIG. 6.6. Test 1. Error $\|\vec{u}-\vec{U}\|$ for different $h$ (top) and $T$ (bottom).

where

$$
a(z, x, y)=10 \frac{d \psi(z, x, y)}{d y}, \quad b(z, x, y)=-10 \frac{d \psi(z, x, y)}{d x}
$$

and

$$
\psi(z, x, y)=(1-\cos (4 \pi z x))(1-x)^{2}(1-\cos (4 \pi z y))(1-y)^{2}
$$



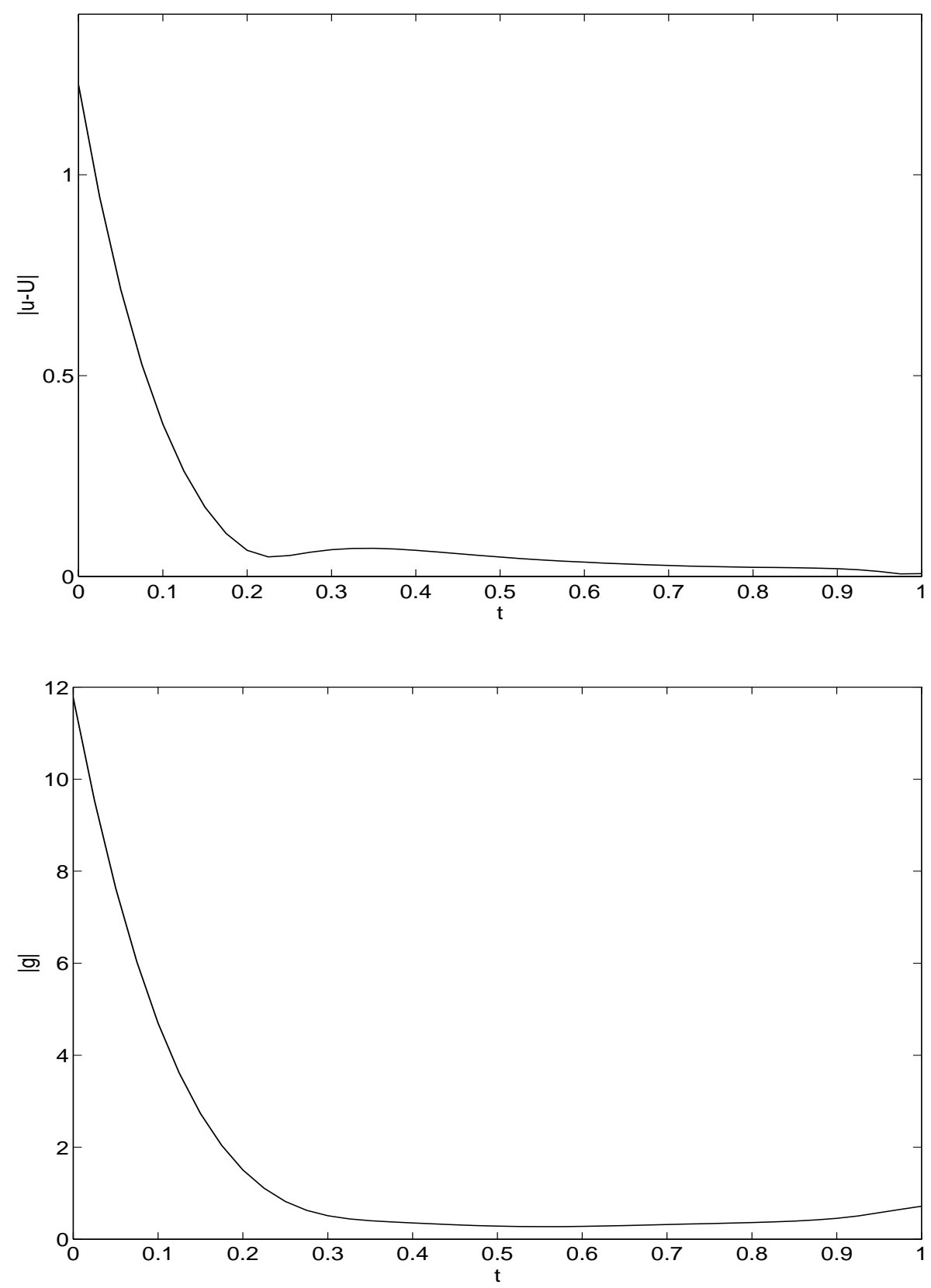

FIG. 6.7. Test 2. Error $\|\vec{u}-\vec{U}\|$ (top) and control norm $\|g\|$ (bottom).

The resulting target velocity is a superposition of two flows: a vortex at the center of the domain with large radius and another vortex with small radius centered in the lower left corner. Each of these flows prevails at different times of the evolution. We set $\alpha=1, \beta=1 / 5000, \gamma=0.5, \Delta t=0.025$, and $h=1 / 16$.

The initial velocity for the controlled flow is

$$
u_{0}(x, y)=(\cos (2 \pi x)-1) \sin (2 \pi y),
$$




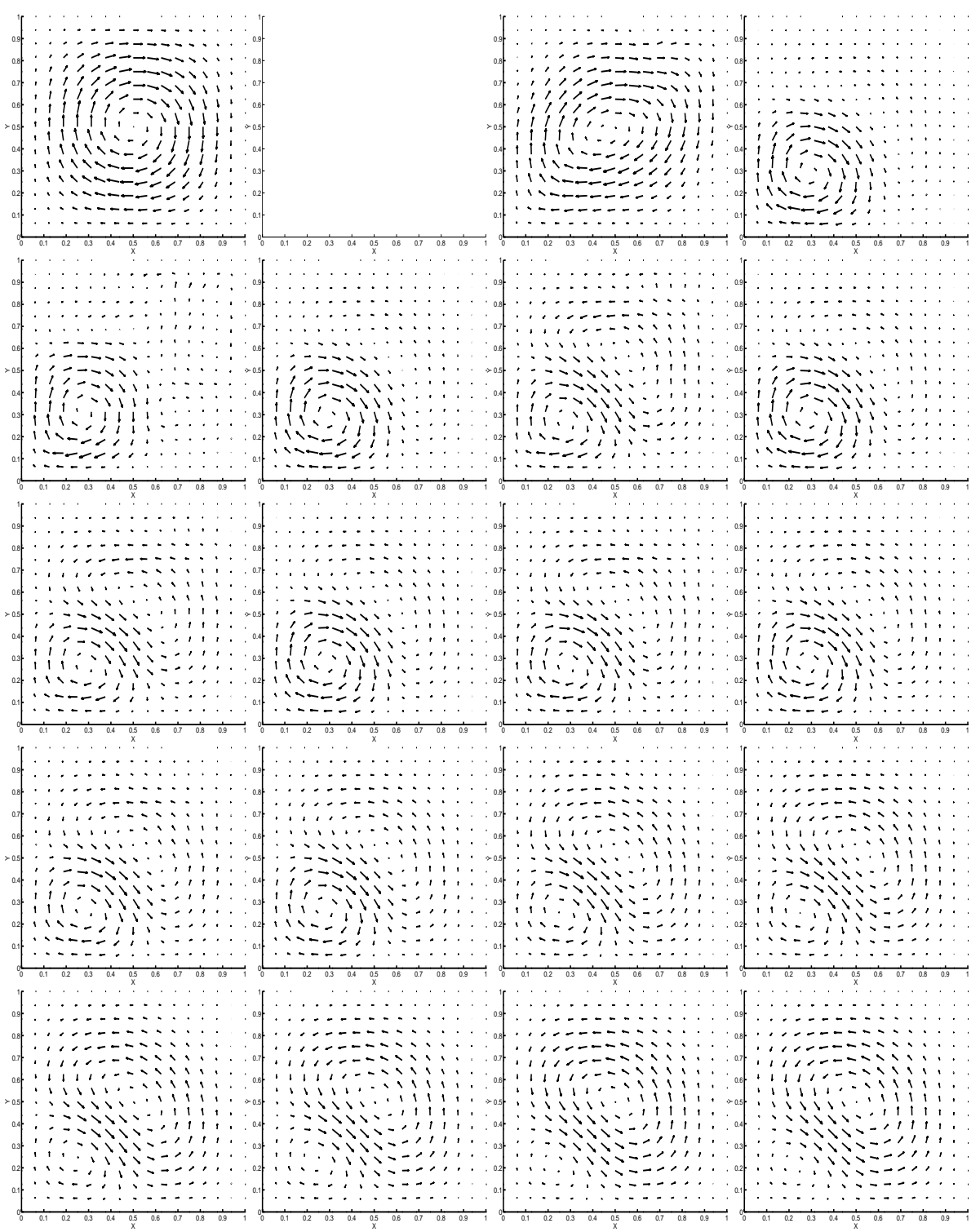

FIG. 6.8. Test 2. Controlled (first and third columns) and target (second and fourth columns) flows at $t=0$ and .1 (first row), $t=.2$ and .3 (second row), $t=.4$ and .5 (third row), $t=.6$ and .8 (fourth row), and $t=.9$ and 1 (fifth row).

$$
v_{0}(x, y)=-(\cos (2 \pi y)-1) \sin (2 \pi x) .
$$

Figure 6.7 shows the error $\|\vec{u}-\vec{U}\|$ between the controlled flow $\vec{u}$ and the target flow $\vec{U}$ and the norm of the control $\|\vec{g}\|$. We can see the error reduces. The evolution is given in Figure 6.2. The controlled fluid is on the left and the target flow is on the right. We can see that by $t=0.5$ we reach a near perfect match. Again, the control works hard at the beginning in order to steer the controlled flow to the target flow and subsequently remains flat. Near $t=T$, the control strength again increases in order to reduce the error. 


\section{REFERENCES}

[1] F. Abergel and R.Temam, On some control problems in fluid mechanics, Theor. Comput. Fluid Dyn., 1 (1990), pp. 303-326.

[2] R. Adams, Sobolev Spaces, Academic Press, New York, London, 1975.

[3] P. Ciarlet, Introduction to Numerical Linear Algebra and Optimization, Cambridge University Press, Cambridge, UK, 1989.

[4] P. Constantin and C. Foias, Navier-Stokes Equations, University of Chicago Press, Chicago, IL, 1989.

[5] A. Fursikov, On some control problems and results related to the unique solution of mixed problems by the three-dimensional Navier-Stokes and Euler equations, Dokl. Akad. Nauk SSSR, 252 (1980), pp. 1066-1070.

[6] A. Fursikov, Control problems and results on the unique solution of mixed problems by the three-dimensional Navier-Stokes and Euler equations, Mat. Sb., 115 (1981), pp. 281-306.

[7] A. Funsikov, Properties of solutions of certain extremum problems related to the Navier-Stokes and Euler equations, Mat. Sb., 117 (1981), pp. 323-349.

[8] V. Girault and P.-A. Raviart, Finite Element Methods for Navier-Stokes Equations, Springer-Verlag, Berlin, New York, 1986.

[9] M. Gunzburger, Finite Element Methods for Incompressible Viscous Flows: A Guide to Theory, Practice, and Algorithms, Academic Press, Boston, MA, 1989.

[10] M. Gunzburger, L. Hou, And T. Svobodny, Analysis and finite element approximation of optimal control problems for the stationary Navier-Stokes equations with Dirichlet controls, RAIRO Modél Math. Anal. Numér., 25 (1991), pp. 711-748.

[11] M. Gunzburger, L. Hou, and T. Svobodny, Analysis and finite element approximation of optimal control for the stationary Navier-Stokes equations with distributed and Neumann controls, Math. Comp., 57 (1991), pp. 123-151.

[12] L. S. Hou AND Y. YAn, Dynamics for controlled Navier-Stokes systems with distributed controls, SIAM J. Control Optim., 35 (1997), pp. 654-677.

[13] L. S. Hou AND Y. YAN, Dynamics and approximations of a velocity tracking problem for the Navier-Stokes flows with piecewise distributed controls, SIAM J. Control Optim., 35 (1997), pp. $1847-1885$.

[14] R. Temam, Navier-Stokes Equations, North-Holland, Amsterdam, 1979. 